Technische Hochschule Wildau

$+$

Brandenburgische

Technische Universität

Cottbus-Senftenberg

$\overrightarrow{\text { Innovation Hub } 13}$

fast track to

T R A N S F E R

Working Paper № 005

2022

Die Flughafenregion Berlin-Brandenburg: Analyse des Status quo als Bestandteil einer Szenarioanalyse zur Zukunft der Region als Wissenschafts- und Innovationsstandort

Frank Hartmann und Dana Mietzner 
fast track to transfer - die Working Paper Series des Innovation Hub 13 ermöglicht es Projektmitarbeiter:innen und Kooperationspartner:innen, sowie weiteren an der Thematik des Wissens- und Technologietransfers interessierten Wissenschaftler:innen und Praktiker:innen, ihre Forschungsergebnisse und praktischen Erfahrungen der wissenschaftlichen Community, sowie einer breiten Öffentlichkeit zur Diskussion zu stellen. Als Instrument zum „fast track to transfer" bietet sie die Möglichkeit, Ergebnisse, Einblicke und Erkenntnisse schnell zu veröffentlichen. Darüber hinaus soll sie den Diskurs mit Akteur:innen aus Wissenschaft, Wirtschaft, Verwaltung, Kultur und Kunst sowie der Zivilgesellschaft fördern, der Information dienen und dazu einladen, sich in die Diskussion um die Zukunft des Wissens- und Technologietransfers einzubringen.

www.innohub13.de/workingpaperseries

Zitationsvorschlag:

Hartmann, Frank/Mietzner, Dana (2022): Die Flughafenregion Berlin-Brandenburg: Analyse des Status quo als Bestandteil einer Szenarioanalyse zur Zukunft der Region als Wissenschafts- und Innovationsstandort. Fast track to transfer (working paper series), No. 005,

DOI: 10.15771/innohub_5

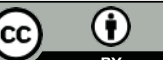

\section{fast track to T R A N S F E R}

Working Paper Series

Technische Hochschule Wildau Hochschulring 1

15745 Wildau

www.th-wildau.de

Brandenburgische Technische Universität Cottbus-Senftenberg Platz der Deutschen Einheit 1 03046 Cottbus www.b-tu.de

Weitere Informationen zum Innovation Hub 13 und zu Transfer finden Sie unter www.innohub13.de

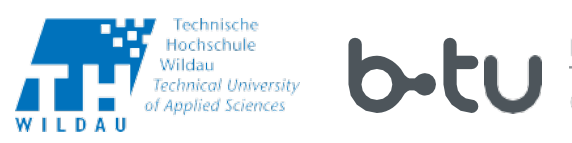

Brandenburgische

Technische Universität Cottbus - Senftenberg
Der "Innovation Hub 13 - fast track to transfer" der Technischen Hochschule Wildau und der Brandenburgischen Technischen Universität Cottbus-Senftenberg gehört zu den 29 ausgewählten Gewinnern der Bund-LänderFörderinitiative „Innovative Hochschule”, ausgestattet mit Mitteln des Bundesministeriums für Bildung und Forschung $\mathrm{BMBF}$ und des Landes Brandenburg. Weitere Informationen finden Sie unter www.innovative-hochschule.de
EINE GEMEINSAME INTIATIVE VON

Innovative Hochschule
Bundesministerium

Bundesminis
für Bildung und Forschun 


\title{
Die Flughafenregion Berlin-Brandenburg: Analyse des Status quo als Bestandteil einer Szenarioanalyse zur Zukunft der Region als Wissenschafts- und Innovationsstandort
}

Hartmann, Frank, Technische Hochschule Wildau, Innovation Hub 13 - fast track to transfer; frank.hartmann@th-wildau.de Mietzner, Dana, Technische Hochschule Wildau, Innovation Hub 13 - fast track to transfer

\begin{abstract}
Internationale Flughäfen sind wichtige Infrastrukturkomponenten mit der Funktion, vielfältige internationale Austauschprozesse zu ermöglichen. Dabei spielen Face-to-Face Kontakte über große Entfernungen hinweg eine Rolle, die für den Austausch vor allem von implizitem Wissen von großer Bedeutung sind. Bei der Realisierung dieser Funktion stehen Flughäfen in Wechselbeziehungen mit zahlreichen regionalen Entwicklungsbedingungen. Sie nehmen diese in Anspruch und entfalten hierbei vielfältige direkte und indirekte Wirkung auf ihr Umfeld. Auf diese Weise entstehen funktionale Räume, die als Flughafenregionen bezeichnet werden. Das vorliegende Working Paper beschreibt den Status quo der Flughafenregion Berlin-Brandenburg. Dem liegt eine Analyse zugrunde, die von einem Regionenverständnis geleitet ist, das system- und akteursbezogene Perspektiven aufweist. Sie geht weiterhin von der gemeinsamen These verschiedener Ansätze aus der Wirtschaftsgeografie und Innovationsforschung aus, die besagen, dass die Bedeutung räumlicher Nähe der Wissenserzeugung und -anwendung sowie des regionalen, interorganisationalen Lernens für Innovationsprozesse vorteilhaft sind. Charakterisiert wird die Flughafenregion deshalb als Wissenschafts- und Innovationsstandort. Gefragt wird darüber hinaus nach Ansätzen für eine Spezialisierung. Im Ergebnis wird deutlich, dass die Region bereits über starke Potenziale in diesen Bereichen verfügt, gegenwärtig eine hohe Ansiedlungsdynamik aufweist, aber noch kein typisches wirtschaftliches Spezialisierungsmuster ausgeprägt hat. Die zukünftige Entwicklung ist diesbezüglich offen. Im weiteren Verlauf der Szenarioanalyse wird daher der Frage nachgegangen, ob sich Spezialisierungspfade entwickeln werden, welche das sein könnten und wie regionale Entwicklungsbedingungen und Akteurskonstellationen beschaffen sein müssten, um einen solchen Profilierungsprozess zu befördern.
\end{abstract}

\section{Keywords}

Flughafenregion, regionale Entwicklungsbedingungen, Wirtschaftsstruktur, Wissenschaftspotenziale 


\section{$\underline{\text { Inhalt }}$}

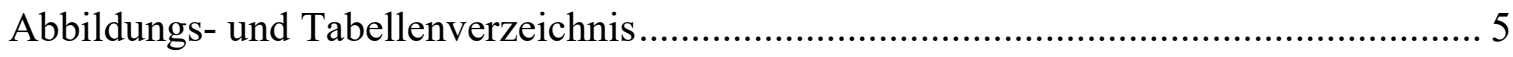

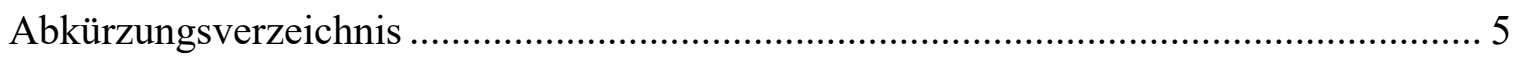

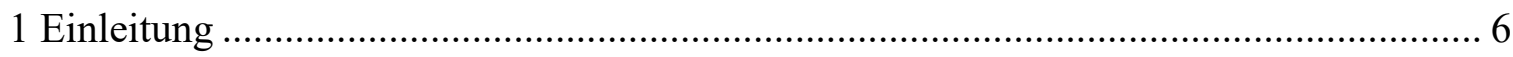

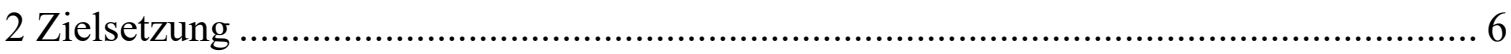

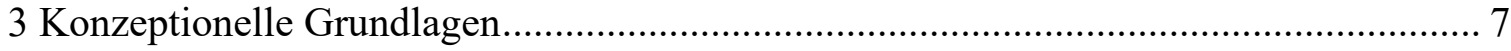

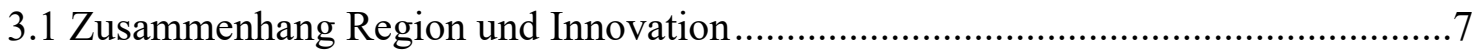

3.2 Zusammenhang Flughafen und Flughafenregion ............................................. 10

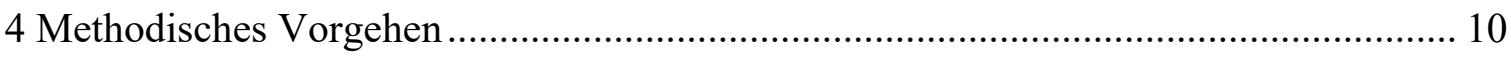

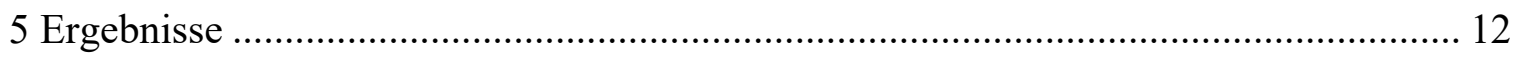

5.1 Bestimmung der Gebietskulisse der Flughafenregion BER ...................................12

5.2 Ausgewählte regionale Entwicklungsbedingungen ............................................13

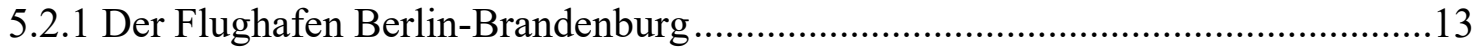

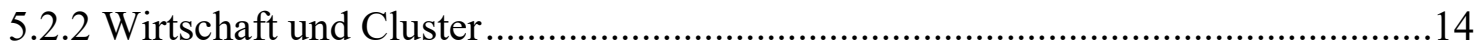

5.2.3 Wissenschaftslandschaft und konzentrierte Technologiestandorte ......................20

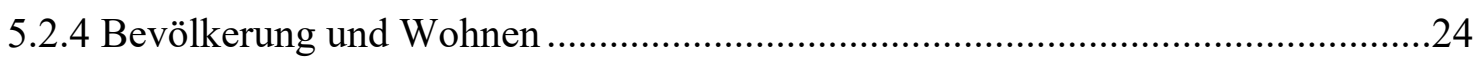

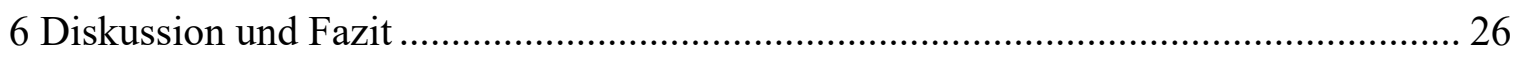

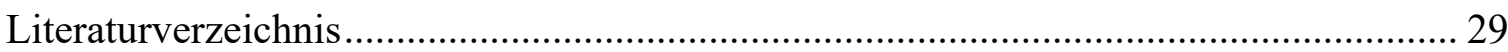




\section{Abbildungs- und Tabellenverzeichnis}

Abbildung 1: Gebietskulisse der Flughafenregion im Rahmen der Szenarioanalyse 12 Abbildung 2: Innere Struktur des Verarbeitenden Gewerbes

Abbildung 3: Innovative, technologieorientierte Unternehmen............................... 19

Tabelle 1: Zuordnung wissenschaftlicher Kompetenzen zu Clustern

\section{Abkürzungsverzeichnis}

Auto-ID Automatische Identifikation und Datenerfassung

BMBF Bundesministerium für Bildung und Forschung

BT Biotechnologie

DLR Deutsches Zentrum für Luft- und Raumfahrt

FB Fachbereich

FBB Flughafen Berlin Brandenburg GmbH

GSK Gemeinsames Strukturkonzept

HTW Hochschule für Technik und Wirtschaft Berlin

IAU île-de-France - Paris Region Urban Planning and Development Agency

IHK Industrie- und Handelskammer

IKT Informations- und Kommunikationstechnologie

IoT Internet of Things

KAG DF Kommunale Arbeitsgemeinschaft Dialogforum Airport Berlin Brandenburg

KMU Kleine und Mittlere Unternehmen

KW Königs Wusterhausen

LBV Landesamt für Bauen und Verkehr

LDS Landkreis Dahme-Spreewald

LOS Landkreis Oder-Spree

MWAE Ministerium für Wirtschaft, Arbeit und Energie

MWFK Ministerium für Wissenschaft, Forschung und Kultur

ÖPNV Öffentlicher Personennahverkehr

PM Potsdam-Mittelmark

RFID Radio-Frequency Identification

RWK Regionaler Wachstumskern

TH Technische Hochschule

TU Technische Universität

USP Unique Selling Proposition

WFBB Wirtschaftsförderung Land Brandenburg GmbH

WFG Wirtschaftsförderungsgesellschaft

ZEW Leibniz-Zentrum für Europäische Wirtschaftsforschung GmbH 


\section{Einleitung}

Mit der Eröffnung des Hauptstadtflughafens Berlin Brandenburg „Willy Brandt“(BER) im Oktober 2020, dem Bau der ersten Gigafactory für Elektroautos von Tesla in Europa in Grünheide und der Microvast-Batteriefabrik in Ludwigsfelde sowie zahlreichen Immobilienentwicklungsvorhaben im engeren Flughafenumfeld sind deutlich Signale für den Beginn einer neuen Phase der Entwicklung der Flughafenregion BER erkennbar. Gebremst wird diese Entwicklung seit Anfang 2020 durch die COVID-19-Pandemie, die ganz besonders die Airlines und Flughäfen, aber auch entsprechende Zulieferbranchen sowie die Kommunen im Flughafenumfeld betrifft. In diese durch große Unsicherheit geprägte Zeit fällt der Entschluss der TH Wildau, eine Szenarioanalyse zur Zukunft der Flughafenregion durchzuführen, um deren Regionalakteur:innen für mögliche Zukünfte zu sensibilisieren und gemeinsam mit ihnen zukunftsbezogene Handlungsoptionen zu erarbeiten. In der Vorbereitungsphase der Szenarioanalyse erfolgten Abstimmungen mit dem Dialogforum BER sowie der für die Fortschreibung des Gemeinsamen Strukturkonzeptes der Flughafenregion beauftragten complan Kommunalberatung GmbH. Gegenstand der Abstimmungen waren die Einbindung der Szenarioanalyse in die geplanten Aktivitäten der Region, eine geeignete Gebietskulisse für die Szenarioanalyse, deren inhaltliche Ausrichtung sowie die Auswahl von Akteursgruppen, die als Beteiligte und/oder Adressaten eingebunden werden sollen. Im vorliegenden Working Paper werden Ergebnisse der Analyse des Status quo der Flughafenregion vorgestellt, die Bestandteil der Vorbereitungsphase der Szenarioanalyse ist. Sie wurde im ersten Quartal 2021 durchgeführt und konnte auf eigenen Vorarbeiten aufbauen (vgl. Mietzner et al. 2020).

\section{$\underline{2 \text { Zielsetzung }}$}

Die Analyse des Status quo dient dem besseren Verständnis der Entwicklungspotenziale und Herausforderungen, denen sich die Flughafenregion gegenübersieht und als Grundlage für die Kommunikation mit Regionalakteur:innen. Darüber hinaus soll sie Orientierung für die Identifizierung derjenigen Faktoren geben, die die zukünftige Entwicklung der Region maßgeblich beeinflussen. Die Identifizierung der Faktoren bildet den nächsten Arbeitsschritt der Szenarioanalyse und soll partizipativ, unter Einbindung von Regionalakteur:innen aus den Bereichen Wissenschaft, Wirtschaft und Kommune erfolgen. Mit der Szenarioanalyse insgesamt soll die Frage beantwortet werden, wie sich die Flughafenregion als Wissenschafts- und Innovationsstandort entwickeln kann, ob sich wirtschaftliche Spezialisierungspfade abzeichnen, welche das sein könnten und wie Regionalakteur:innen mit einer möglichen Spezialisierung umgehen sollten. Diese Fragestellung hat auch bereits den Fokus der Status quo Analyse beeinflusst, der auf Potenzialen (im Sinne von Ressourcen und Kompetenzen) in den Bereichen Wissenschaft und Wirtschaft liegt, ohne andere regionale Entwicklungsbedingungen und entsprechende Zusammenhänge aus dem Blick zu verlieren. 


\section{Konzeptionelle Grundlagen}

Nachfolgend werden ausgewählte konzeptionelle Grundlagen skizziert, die bei der Formulierung der Fragestellung der Szenarioanalyse eine Rolle gespielt haben und die orientierend für die Analyse des Status quo der Flughafenregion sowie das entsprechende methodische Vorgehen waren.

\subsection{Zusammenhang Region und Innovation}

Unter Region wird nach Wiechmann ein sozio-ökonomischer Verflechtungsraum verstanden, der sich durch die gegenseitige Wahrnehmung der Akteure definiert, die in ihm agieren (vgl. Wiechmann 2000: 181). Diese Auffassung erweiternd geht Voß davon aus, dass Regionen unterschiedlich dimensionierte und differenzierte natürliche, ökonomische und kulturelle Arbeits- und Lebensräume sind. Sie werden durch die Beziehungen und Interessen privater und öffentlicher Akteure aus Wirtschaft, Wissenschaft, Bildung und Politik gestaltet und verfügen über eine kritische Masse an endogenen Entwicklungspotenzialen sowie an Zugängen zu außerhalb liegenden Umwelten (vgl. Voß 2002: 28-29). Neuere Perspektiven auf die Regionalentwicklung sind u. a. durch die Annahme der kommunikativen Konstruktion von Räumen gekennzeichnet. In diesem Verständnis werden Räume als relationale, von handelnden Subjekten ausgehandelte und gestaltete Konstrukte verstanden. Im Mittelpunkt dieser Ansätze steht die Frage, wie regionales Wissen kommunikativ entsteht, vermittelt, verhandelt und anschlussfähig gemacht wird (vgl. Christmann 2015: 7-8). Beide Ansätze sind für die Szenarioanalyse zur Flughafenregion relevant. Für die Analyse des Status quo spielen zunächst die endogenen Potenziale eine Rolle. Die endogenen Potenziale werden durch regionale Entwicklungsbedingungen beschrieben, die unter Zuhilfenahme ausgewählter Indikatoren charakterisiert werden können. $\mathrm{Zu}$ den regionalen Entwicklungsbedingungen zählen beispielsweise die technische und soziale Infrastruktur einer Region, ihre Wirtschaftspotenziale und -struktur oder auch ihre Siedlungsstruktur (vgl. Voß 2002: 29). Dabei ist zu berücksichtigen, dass es nicht allein das Vorhandensein/Nichtvorhandensein einzelner regionaler Entwicklungsbedingungen ist, was über erfolgreiche Regionalentwicklung entscheidet. Es geht vielmehr um die Passfähigkeit der regionalen Entwicklungsbedingungen zu den Entwicklungszielen einer Region. Dabei sind die quantitative und qualitative Verfügbarkeit regionaler Entwicklungsbedingungen ebenso zu berücksichtigen wie ihre wechselseitigen Beziehungen im Systemzusammenhang (vgl. Voß 2002: 28-29). „In diesem Kontext lassen sich auch erst sinnvoll regionale Disparitäten, Defizite, Stärken und Schwächen bestimmen...] "(ebd.). Voraussetzung für eine erfolgreiche Regionalentwicklung werden damit die Wahrnehmung und Bewertung der Zusammenhänge zwischen Anforderungen und Beschaffenheit sowie das entsprechende Handeln von Regionalakteuren: Es gilt, die systembezogene und die akteursbezogene Perspektive zu verbinden. Basierend auf diesem Verständnis lässt sich auch der Zusammenhang zur 
Innovationsproblematik herstellen: Sind regionale Entwicklungsbedingungen nicht passfähig zueinander und/oder zu bestimmten regionalen Entwicklungszielen, können regionale Problemlagen entstehen, die darauf bezogene Problemlösungsprozesse (Innovationen) herausfordern und orientieren (vgl. Voß 2002: 30).

Darüber hinaus wird der Zusammenhang von Regionalentwicklung und Innovation seit vielen Jahren in Konzepten der Wirtschaftsgeografie und Regionalökonomie, aber auch der Innovationsforschung diskutiert. Eine umfangreiche Übersicht über die Konzepte findet sich beispielsweise bei Koschatzky (2001) und Barjak (2011). Im Rahmen des vorliegenden Forschungsberichtes werden lediglich diejenigen Aspekte ausgewählter Konzepte aufgegriffen, die für die Analyse des Status quo der Flughafenregion von Relevanz sind.

Im Konzept des Innovationsmilieus ergänzen informelle und implizite Beziehungen die formellen Akteursbeziehungen in einer Region bei der Gestaltung von Innovationsprozessen (vgl. Camagni/Capello 2002: 18). Aufgrund räumlicher Nähe werden interorganisationale Lernprozesse unterstützt, Unsicherheiten in Innovationsprozessen können reduziert und Koordinationskosten gesenkt werden (vgl. Camagni/Capello 2002: 15).

Das Clusterkonzept von Porter (1998) hebt die regionale Wettbewerbsfähigkeit als Stimulus für Innovationen hervor und betont die besondere Rolle wissenschaftlichtechnischen Wissens, des spezifischen Knowhows sowie von Innovationen für die Entwicklung von Clustern in Regionen (vgl. Porter 1998: 78).

Im Konzept des regionalen Innovationssystems interagieren unterschiedliche regionale Akteurstypen bezogen auf Innovation, wobei Wissenschaft und Forschung zentrale Akteure sind. Regionale Innovationssysteme erlangen ihre Wirkmächtigkeit durch ihre systemische Ordnung, die kollektiver Art ist und auf Vertrauen, Verlässlichkeit, Austausch und kooperativer Interaktion beruht (vgl. Cooke et al. 1997: 490).

Im Triple Helix Modell steht das Zusammenwirken von Wissenschaft, Wirtschaft und Staat im Fokus, mit der besonderen Fähigkeit von Netzwerken, mit internen und externen Dynamiken umzugehen. Triple Helix Regionen sind in der Lage, technologische Paradigmenwechsel zu gestalten und neue Technologien und Geschäftsmodelle hervorzubringen, indem unterstützende Innovationsstrukturen geschaffen werden (vgl. Etzkowitz/Zhou 2008: 124). Konstitutiv sind dabei Wissensräume, Konsensräume und Innovationsräume (vgl. Etzkowitz/Zhou 2008: 125). Im Unterschied zum Konzept des Innovationssystems spielt im Triple-Helix-Konzept die unternehmerische Universität eine zentrale Rolle, die Interaktion der Akteure wird als Interaktion institutioneller Sphären verstanden. Statt Selbstorganisation des Systems spielen Innovationsorganisatoren eine wichtige Rolle (vgl. Etzkowitz/Zhou 2008: 125). 
Im Konzept Smart Specialisation entsteht die regionale Entwicklungsdynamik aus einem Bottom-up getriebenen unternehmerischen Entdeckungsprozess bezogen auf neue, übergreifende Geschäftsbereiche im Zusammenhang mit F\&E und Innovation (vgl. Foray/Goenaga 2013: 6). In diesem experimentellen, kollektiven Prozess, in den unterschiedliche Regionalakteure eingebunden sind (vgl. Kempton et al. 2014: 17), werden Konzentrationsprozesse angeregt und Wissensspillovers (im Sinne von Übertragungseffekten) regional genutzt. Dieser Prozess ist nicht Top-down getrieben, kein Planungsprozess im traditionellen Verständnis, sondern im Mittelpunkt stehen innovative Firmen, Entdecker und Erfinder sowie anerkannte Wissenschaftler:innen (vgl. Foray 2013: 5). Der Prozess der smarten Spezialisierung knüpft an endogene Entwicklungspotenziale einer Region an, vor allem im Sinne von vorhandenen Fähigkeiten oder Kompetenzen, wobei besonders auf nichtkodifiziertes, lokales Wissen Bezug genommen wird (vgl. Foray/David/Hall 2009: 2). Gleichzeitig ist der Zutritt neuer Akteure zu den identifizierten Geschäftsbereichen von Bedeutung. Smarte Spezialisierung resultiert letztlich in strukturellem Wandel, der sich in der Form der Modernisierung bestehender Strukturen, der Diversifikation oder auch der Transformation traditioneller Wirtschaftsbereiche äußern kann. Er ist nicht allein auf die hoch dynamischen Wirtschaftsbereiche einer Region gerichtet, sondern eröffnet auch traditionellen Wirtschaftsbereichen die Chance zur Spezialisierung (vgl. Foray/Goenaga 2013: 7). Smarte Spezialisierung fungiert als ein innovationspolitisches Konzept, um Regionen bei der Identifizierung von Entwicklungsschwerpunkten zu unterstützen und solche Interventionen anzuregen, die Ressourcen und Kompetenzen auf diese Schwerpunkte ausrichten (vgl. Vezzani et al. 2017: 5). Am Beginn einer auf Smarte Spezialisierung ausgerichteten Analyse stehen nach Vezzani et al. die Ressourcen und Kompetenzen der Wissenschaftseinrichtungen und der Industrie sowie Ansätze einer wirtschaftlichen Spezialisierung. Die Dynamik unternehmerischer Aktivitäten in vorhandenen Wirtschaftsbereichen sowie neu entstehende Sektoren sind in einer solchen Analysephase zu berücksichtigen (vgl. ebd.: 6). Die auf die regionalen Entwicklungsbedingungen ausgerichteten Analysen sollten dann um externe und auf die Zukunft gerichtete Perspektiven ergänzt werden. Letzteres schließt auch Foresightansätze ein (vgl. ebd.: 7).

Allen Konzepten gemeinsam ist die Bedeutung von räumlicher Nähe der Wissenserzeugung und -anwendung sowie des regionalen, interorganisationalen Lernens für Innovationsprozesse. Dabei spielen Wissenschafts- und Forschungseinrichtungen sowie technologisch-wirtschaftliche Innovationsprozesse nach wie vor eine zentrale Rolle. In der Analyse des Status quo wird insbesondere Bezug auf das Konzept Smart Specialisation Bezug genommen. Die räumliche Nähe und Interaktion wissenserzeugender und -anwendender Akteure ist vor allem für die Bestimmung der Gebietskulisse und das Verständnis/Selbstver- 
ständnis der Region von Bedeutung. Die Annahmen zur Beschaffenheit regionaler Entwicklungsbedingungen und deren Systemzusammenhang orientieren die Analyse inhaltlich sowie die Logik der Ergebnisdarstellung.

\subsection{Zusammenhang Flughafen und Flughafenregion}

Internationale Flughäfen sind wichtige strategische Infrastrukturen, die die Funktion haben, die Anschlussfähigkeit ganzer Nationen und Regionen an vielfältige globale Austauschprozesse zu gewährleisten (vgl. IAU 2018: 5). Sie ermöglichen den Austausch von Wissen über große Entfernungen hinweg. Hierbei spielt vor allem das implizite Wissen eine Rolle, das in ihrem Umfeld generiert wird und ein wichtiger Innovationstreiber (vgl. Piirainen/Tanner/Alkaersig 2017: 291) ist. Aus diesem Grund können Flughafenregionen auch als Hotspots der Wissensökonomie bezeichnet werden, die im Kern von High-Tech Unternehmen und wissensintensiven Dienstleistern unterschiedlichen Typs in räumlicher Nähe gebildet wird (vgl. Kujath/Schmidt 2007: 9-11).

Bei der Realisierung dieser Austauschs- und Kommunikationsfunktion stehen Flughäfen in Wechselbeziehungen zu zahlreichen Entwicklungsbedingungen in ihrem jeweiligen Umfeld (vgl. Abschnitt 3.1). Sie nutzen und fordern vor allem Verkehrsinfrastrukturen, Arbeitskräfte, Flächen und Wirtschaftsstrukturen. Damit verbunden, entfalten sie vielfältige direkte und indirekte Wirkung auf ihr Umfeld (vgl. Baum et al. 2005: 80). Es entstehen funktionale Räume, die als Flughafenregionen bezeichnet werden (vgl. Droß/Thierstein 2011: 27). Je nach Flughafentyp und geografischem Kontext können diese Flughafenregionen unterschiedliche Gebietskulissen aufweisen (vgl. IAU 2018: 5). Sie entwickeln sich, wie andere Regionen auch, über längere Zeiträume hinweg und prägen in differenzierter Art und Weise Profile aus.

Zusammenfassend lässt sich festhalten, dass Flughafenregionen sich von anderen Regionen durch ihre Funktionalität unterscheiden. Sie verfügen in der Regel über Entwicklungspotenziale im Sinne von Kompetenzen und Ressourcen, die deren Innovationsfähigkeit befördern. Diesen Kompetenzen und Ressourcen wird in der weiteren Analyse des Status quo der Flughafenregion BER etwas detaillierter nachgegangen.

\section{Methodisches Vorgehen}

Das methodische Vorgehen der Status quo Analyse beruht auf der Kombination qualitativer und quantitativer Methoden. Zu beantworten war die Frage, wie die Flughafenregion als Wissenschafts- und Innovationsstandort derzeit aufgestellt ist und ob sich Ansätze für eine Spezialisierung erkennen lassen. Ausgehend von der Bestimmung der Gebietskulisse der Region, die sich an die Definition im „Gemeinsamen Strukturkonzept für die Flughafenregion“" aus dem Jahr 2007 anlehnt (vgl. GSK 2007) und diese erweitert, wurden ausgewählte regionale Entwicklungsbedingungen analysiert. Im Fokus standen die 
Wirtschaftsstruktur, mit dem Schwerpunkt innovative, technologieorientierte Unternehmen, Wissenschaftspotenziale, Bevölkerungsentwicklung und Wohnen.

Unter innovativen, technologieorientierten Unternehmen werden solche Unternehmen verstanden, deren Leistungsangebote auf neuen technologischen Ideen und Forschungsergebnissen basieren und in denen die Entwicklung und Vermarktung neuer Technologien wesentlicher Bestandteil der Unternehmensstrategie ist (vgl. Wagner und Schultz, 2011:30). Sie bilden einen Teil der innovativen Unternehmen/Innovatoren, also derjenigen Unternehmen, die innerhalb eines zurückliegenden Zeitraumes von drei Jahren zumindest eine Produkt- oder Prozessinnovation eingeführt haben (ZEW, 2019:14), wobei bei dieser Analyse vor allem nach Unternehmen mit Produktinnovationen gesucht wurde. Voraussetzung, um als Unternehmen in diese Auswahl aufgenommen zu werden, war darüber hinaus die eigene Durchführung von Forschung und/oder Entwicklung bzw. die Beteiligung daran. Kriterien hierfür waren die Inanspruchnahme von Forschungs- und Entwicklungsfördermaßnahmen auf Ebene des Bundes und des Landes, Innovationskooperationen sowie die Nominierung bzw. das Gewinnen von Innovationspreisen.

Für den Brandenburger Teil der Flughafenregion wurden die Unternehmen in einem sehr detaillierten Suchprozess identifiziert. Er umfasste die Recherche im Förderkatalog der Bundesregierung (Projekte ab 2010), die Sichtung von Übersichten zu Förderungen im Land Brandenburg mit Mitteln des ESF (Brandenburger Innovationsfachkräfte [BIF], Innovationsassistenten) (2014-2020) bzw. des EFRE, Interventionskategorie Forschungsund Innovationsprozesse in KMU sowie Fortgeschrittene Unterstützungsdienste für KMU und KMU-Zusammenschlüsse (2014-2020), die Übersicht zu Innovationspreisen ab 2010, Branchenverzeichnisse der Gemeinden und Webseiten von Technologiezentren sowie Wirtschaftsförderungsgesellschaften.

Für den Berliner Teil der Flughafenregion wurden die Unternehmen basierend auf den Webseiten der Technologie- und Gründerzentren sowie der Wirtschaftsförderungen der Stadtbezirke ermittelt. Die auf diese Weise identifizierten Unternehmen wurden den Clustern der gemeinsamen Innovationsstrategie der Länder Berlin-Brandenburg (vgl. WFBB o. J.) zugeordnet.

Wissenschaftspotenziale in beiden Teilen konnten durch Internetrecherchen ermittelt werden. Die Analyse der Wirtschaftsstruktur, der Bevölkerungsentwicklung sowie des Bereichs Wohnen erfolgte unter Nutzung statistischer Daten der Länder Berlin und Brandenburg. Auch sie erfolgte für die definierte Gebietskulisse der Flughafenregion, wofür insbesondere die Daten einzelner Kommunen, Stadtbezirke und Landkreise genutzt wurden. Bei der Analyse des Status quo wurde auch auf Interviews mit Regionalakteuren zurückgegriffen, die im Rahmen einer Vorstudie geführt wurden (vgl. Mietzner et al. 2020). Hierauf basieren Einschätzungen zum Gewerbeflächenpotenzial, zur Rolle von 
Entwicklungsachsen oder auch zur Zusammenarbeit von Kommunen.

\section{Ergebnisse}

Im Folgenden werden die Ergebnisse der Status quo Analyse dargestellt, beginnend mit der Festlegung der Gebietskulisse der Flughafenregion BER, gefolgt von der Beschreibung regionaler Entwicklungsbedingungen anhand ausgewählter Indikatoren. Den Abschluss bildet ein Fazit zum methodischen Vorgehen und seinen Ergebnissen.

\subsection{Bestimmung der Gebietskulisse der Flughafenregion BER}

Die der Status quo Analyse zugrundeliegende Gebietskulisse der Flughafenregion umfasst die drei Berliner Stadtbezirke Tempelhof-Schöneberg, Neukölln und Treptow-Köpenick. Zum Brandenburger Teil zählen Blankenfelde-Mahlow, Eichwalde, Fürstenwalde, GosenNeu Zittau, Großbeeren, Grünheide, Königs Wusterhausen, Lübben, Luckenwalde, Ludwigsfelde, Mittenwalde, Rangsdorf, Schönefeld, Schulzendorf, Teltow, Wildau und Zeuthen (vgl. Abbildung 1).

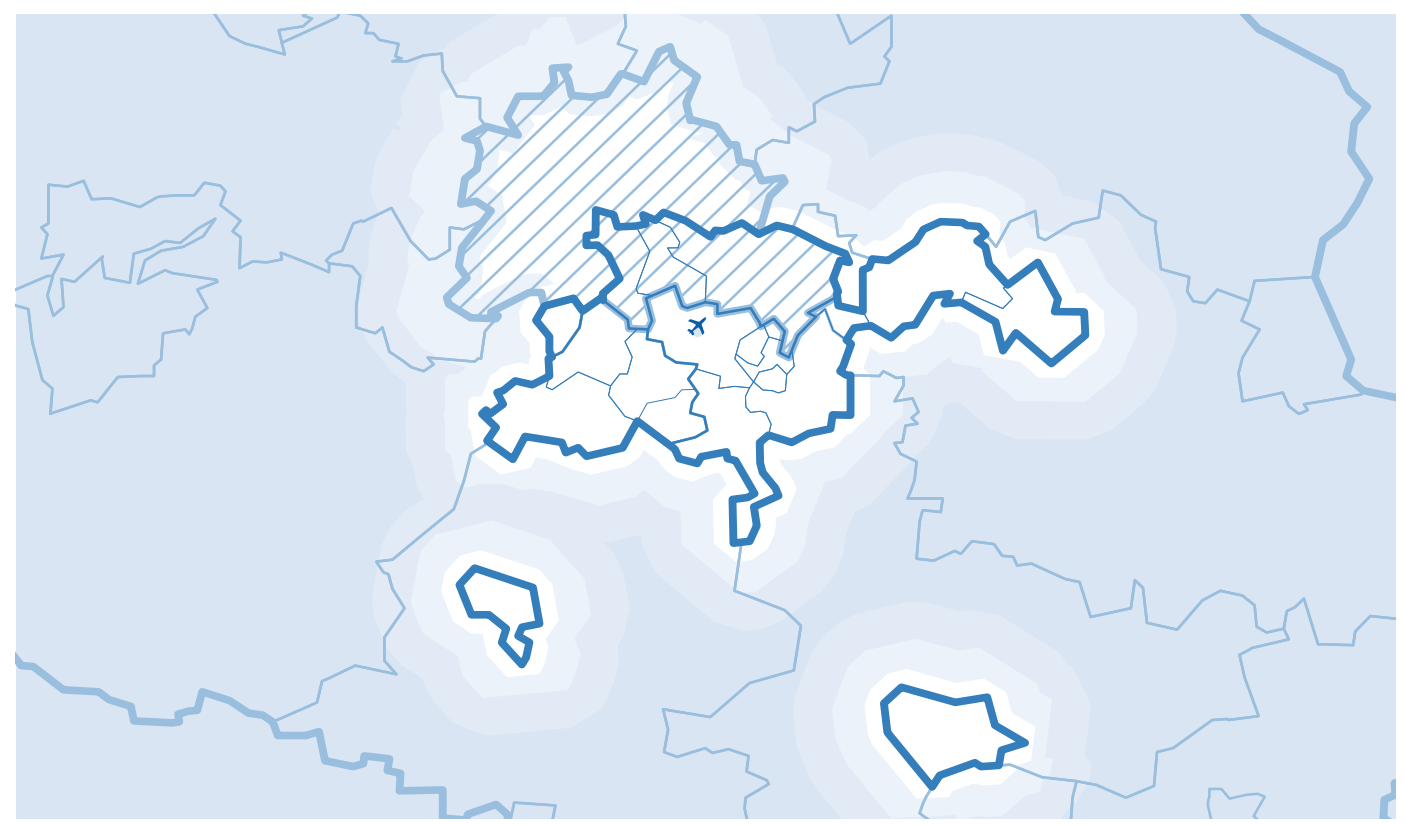

Abbildung 1: Gebietskulisse der Flughafenregion im Rahmen der Szenarioanalyse.

In Anbetracht diverser Entwicklungstendenzen in den letzten Jahren und unter Berücksichtigung des Analyseschwerpunktes „Potenziale in Wissenschaft und Wirtschaft“ wurde im Vergleich zur Gebietskulisse des GSK eine Erweiterung der Flughafenregion um einzelne Städte und Gemeinden vorgenommen. Hierbei handelt es sich um die beiden 
regionalen Wachstumskerne Fürstenwalde und Luckenwalde, die sich in den letzten Jahren wirtschaftlich sehr positiv entwickelt haben und in denen die Technische Hochschule Wildau gemeinsam mit der Universität Viadrina bzw. der Fachhochschule Potsdam Präsenzstellen unterhalten. Beide Wachstumskerne liegen auf wichtigen Entwicklungsachsen der Region und sind innerhalb einer Isochrone von maximal 45 Minuten ausgehend vom Flughafen zu erreichen. Hinzu kommt die Kreisstadt Lübben mit sehr guten Bedingungen für qualitativ hochwertiges Wohnen und Arbeiten im Grünen sowie als potenzieller Ansiedlungsstandort für Wissenschaftseinrichtungen des Standorts Adlershof. Auch die verkehrliche Anbindung von Lübben an den Flughafen wird als gut eingeschätzt. Schließlich sollte auch Grünheide als zukunftsträchtiger Standort der Gigafabrik von Tesla mit Flughafennähe Berücksichtigung in der Analyse des Status quo finden.

\subsection{Ausgewählte regionale Entwicklungsbedingungen}

Nachfolgend werden für die Flughafenregion bestimmende regionale Entwicklungsbedingungen anhand ausgewählter Indikatoren beschrieben, anknüpfend an die Aussagen $\mathrm{zu}$ regionalen Entwicklungsbedingungen und deren Systemzusammenhängen im konzeptionellen Teil (vgl. Abschnitt 3.1). Der Fokus liegt auf den Bereichen Wirtschaft und Wissenschaft. Eine aktuelle und detaillierte Analyse weiterer regionaler Entwicklungsbedingungen findet sich im fortgeschriebenen „Gemeinsamen Strukturkonzept aus dem Jahr 2021“ (vgl. Dialogforum Airport Berlin-Brandenburg 2021).

\subsubsection{Der Flughafen Berlin-Brandenburg}

Eine wichtige regionale Entwicklungsbedingung der Flughafenregion ist der Flughafen selbst, in seiner Beschaffenheit und Funktionalität. Der Flughafen BER ging nach über 20 Jahren Planung und Bau sowie mehrfach verschobenen Eröffnungsterminen am 25. Oktober 2020 in Betrieb und vereint nun als Berliner Hauptstadtflughafen die Kapazitäten der ehemaligen Flughäfen Schönefeld, Tegel und Tempelhof. Eine ausführlichere Historie des BER findet sich auf der Webseite des Flughafens (vgl. Berlin-Airport o. J. a).

Der BER rangiert vom Passagieraufkommen her auf Platz 13 der größten europäischen Flughäfen und Platz 3 der größten deutschen Flughäfen hinter Frankfurt und München (vgl. Flughafendetails 2021). Seine Eröffnung Ende Oktober 2020 fiel in die Zeit des Höhepunktes der zweiten Welle der COVID-19-Pandemie, die massive Auswirkungen auf den Flugbetrieb und den Flughafen hatte. Die Passagierzahlen sind im Jahr 2020 eingebrochen: Betrugen sie im Jahr 2019 noch knapp 36 Millionen Passagiere, lag diese Zahl im Jahr 2020 bei rund neun Millionen Passagieren, was einen Rückgang um 74,5 Prozent bedeutet (vgl. Berlin-Airport o. J. b). Für den Betrieb des BER folgt daraus, dass das Terminal 2 vorerst nicht in Betrieb genommen wird und das Terminal 5 geschlossen ist. Aufgrund der geringen Passagierzahlen geht die Flughafengesellschaft in den nächsten Jahren von erheblichen Umsatzverlusten aus. Sie rechnet für das Jahr 2021 mit etwa 10,7 Millionen Passagieren und geht davon aus, dass das Niveau des Aufkommens des Jahres 
2019 wahrscheinlich erst in fünf Jahren wieder erreicht werden kann (vgl. Berlin-Airport 2021). Um die negativen finanziellen Auswirkungen dieses Rückgangs zu kompensieren, sollen lediglich unbedingt notwendige Investitionen getätigt und Betriebskosten geringgehalten werden (vgl. Berlin-Airport 2021). Die Flughafengesellschaft hat eine Teilentschuldung erbeten, und der Aufsichtsrat hat einen Businessplan zur finanziellen Restrukturierung unterstützt (vgl. Berlin-Airport 2021). „Das Konzept sieht vor, dass die Gesellschafter der FBB ab 2022 in fünf jährlichen Teilschritten insgesamt knapp 1,9 Milliarden Euro zur Verfügung stellen. Davon sind 1,1 Milliarden Euro zur Teilentschuldung der Flughafengesellschaft und rund 800 Millionen Euro als Liquiditätshilfe vorgesehen." (vgl. Berlin-Airport 2021).

Damit sind für die Entwicklung in den nächsten Jahren limitierende Rahmenbedingungen gesetzt, die Konsequenzen für die gesamte Flughafenregion haben. Das betrifft die Arbeitskräftesituation am Flughafen, Investitionsvorhaben auf dem Gelände des Flughafens sowie aufgrund der fehlenden Kaufkraft von Passagieren oder verringerter Auftragsvergabe der Flughafenunternehmen nicht wie erwartet eintretende Effekte (vgl. Baum et al. 2005: 80).

Unabhängig von der gegenwärtig schwierigen Situation, gibt es für den BER einen Masterplan zum Ausbau bis auf eine Kapazität von 55 Millionen Passagieren/Jahr (vgl. Berlin-Airport o. J. c). In einer ersten Stufe ist der Ausbau des Terminals 2 mit sechs Mio. Passagieren/Jahr, des Terminals 1 mit einer maximalen Kapazität bis zu 27 Mio. Passagiere/Jahr und des Terminals 5 mit acht bis zehn Mio. Passagieren/Jahr geplant. Die Gesamtkapazität betrüge in dieser Ausbaustufe bis zu 43 Mio. Passagieren/Jahr. In einer zweiten Ausbaustufe soll das neue Terminal 3 bis Ende der 2020er Jahre das alte Terminal 5 ablösen, mit einer am Bedarf orientierten Kapazität bis zu 15 Mio. Passagieren/Jahr. Die Gesamtkapazität betrüge dann 48 Mio. Passagier/Jahr. In einer dritten Stufe ist der Bau des Terminals 4 vorgesehen, mit einer Kapazität bis zu sechs Mio. Passagieren/Jahr und eine Erweiterung des bisherigen Terminals 1, so dass eine Gesamtkapazität von 55 Mio. Passagieren/Jahr entstünde (vgl. Berlin-Airport o. J. c).

Insofern ist der Flughafen BER in Abhängigkeit von der zukünftigen Nachfrage und der Funktionsfähigkeit des Geschäftsmodells flexibel erweiterbar, was es bei der Entwicklung von Zukunftsprojektionen im Rahmen der Szenarioanalyse zu berücksichtigen gilt.

\subsubsection{Wirtschaft und Cluster}

Anknüpfend an die Bedeutung der regionalen Wirtschaftsstruktur als Anknüpfungspunkt für eine auf Wissenschaft und Technologie beruhende Spezialisierung im Konzept „Smart Specialisation“" werden im Folgenden einige Merkmale der Wirtschaftsstruktur der Flughafenregion BER beschrieben. Die innovativen, technologieorientierten Unternehmen stehen hierbei im Mittelpunkt. Sie sind es vor allem, die anwendungsnahe Ergebnisse aus 
Wissenschaft und Forschung aufgreifen, um Spezialisierungspfade im Sinne von Foray zu entdecken. „Indeed, the entrepreneurial discovery that drives the process of smart specialisation is not simply the advent of an innovation but the deployment and variation of innovative ideas in a specialised area that generate knowledge about the future economic value of a possible direction of change" (Foray 2013: 5).

Die Wirtschaftsstruktur nach Wirtschaftszweigen der NACE-Klassifikation zeigt für die Flughafenregion insgesamt 51.470 aktive Unternehmen im Jahr 2018, von denen 41.309 im Berliner Teil (ca. 80 Prozent) und 10.161 im Brandenburger Teil (ca. 20 Prozent) angesiedelt sind (vgl. StatIS-BBB o. J.). Mit 8.959 Unternehmen nimmt der Wirtschaftszweig ,Erbringung von freiberuflichen, wissenschaftlichen und technischen Dienstleistungen " den ersten Rang ein, der auch im Berliner Teil der Flughafenregion nach der Anzahl der Unternehmen an erster Stelle liegt (vgl. StatIS-BBB o. J.). Im Brandenburger Teil der Region rangiert dieser Wirtschaftszweig hingegen auf Rang 3. Es folgen die Wirtschaftszweige „Handel“ und „Baugewerbe“, die in Deutschland insgesamt die Ränge eins und drei einnehmen (vgl. Statista 2021a). Das Verarbeitende Gewerbe, mit einem i.d.R. hohen Anteil innovativer Unternehmen und dem höchsten Umsatz aller Wirtschaftsbereiche in Deutschland (ebd.), hat nach der Anzahl der Unternehmen in beiden Teilen der Flughafenregion einen Anteil von 4,3 Prozent, der im Vergleich zu Deutschland insgesamt mit knapp sieben Prozent etwas geringer ist (ebd.). Der Anteil des Verarbeitenden Gewerbes liegt im Brandenburger Teil der Flughafenregion mit 5,8 Prozent höher als im Berliner Teil mit 3,8 Prozent (vgl. StatIS-BBB o. J.).

Auf der Ebene der Wirtschaftszweige insgesamt sind Differenzierungen struktureller Art jedoch gering. Nimmt man die großen Unternehmen mit über 250 Mitarbeiter:innen in den Blick, zeigen sich schon deutlicher Unterschiede. So gab es im Jahr 2018 in der Flughafenregion insgesamt 149 große Unternehmen. Davon entfallen 101 Unternehmen auf den Berliner Teil und 48 Unternehmen auf den Brandenburger Teil (vgl. StatIS-BBB o. J.). Auf dieser Betrachtungsebene haben die Unternehmen des Verarbeitenden Gewerbes auch einen deutlich höheren prozentualen Anteil an der Gesamtzahl der Unternehmen. Er liegt für die Flughafenregion insgesamt bei rund 17 Prozent und ist im Berliner Teil mit zehn Prozent etwas größer als im Brandenburger Teil mit sieben Prozent. Das Verarbeitende Gewerbe liegt damit an erster Stelle der Wirtschaftszweige, gleichauf mit dem Gesundheits- und Sozialwesen. Große Unternehmen im Wirtschaftszweig der Erbringung von freiberuflichen, wissenschaftlichen und technischen Dienstleistungen hingegen finden sich nur im Berliner Teil der Flughafenregion. Weiterhin ist hervorzuheben, dass im Brandenburger Teil der Anteil des Wirtschaftszweiges Verkehr und Lagerei größer ist als im Berliner Teil. Hierbei handelt es sich um den einzigen Wirtschaftsbereich, für den das zutrifft.

Auf regionaler Betrachtungsebene zeigt die Verteilung der großen Unternehmen im Berliner Teil mit 56 Unternehmen einen Schwerpunkt im Stadtbezirk Tempelhof- 
Schöneberg, im Brandenburger Teil finden sich die meisten großen Unternehmen der Flughafenregion in Fürstenwalde (9 Unternehmen) und Schönefeld (7 Unternehmen). Davon gehören in Schönefeld allein fünf große Unternehmen zum Wirtschaftszweig Verkehr und Lagerei.

Geht man in der strukturellen Differenzierung einen Schritt weiter und betrachtet die innere Struktur des Verarbeitenden Gewerbes (vgl. StatIS-BBB o. J.) ist erkennbar, dass die meisten Unternehmen in den Bereichen „Herstellung von Metallerzeugnissen“, „Herstellung von sonstigen Waren“ sowie „Herstellung von Nahrungs- und Futtermitteln“ aktiv sind (vgl. Abbildung 2). Deutliche Unterschiede gibt es in der Verteilung der Unternehmen zwischen dem Berliner und dem Brandenburger Teil der Flughafenregion. Während die Bereiche „Druckerzeugnisse“, „Datenverarbeitungsgeräte“ sowie „Nahrungsund Futtermittel“ im Berliner Teil überrepräsentiert sind, ist die Anzahl der Unternehmen im Bereich „Reparatur und Installation von Maschinen und Anlagen“ nahezu gleich verteilt, obwohl im Berliner Teil insgesamt mehr als doppelt so viele Unternehmen im Verarbeitenden Gewerbe aktiv sind wie im Brandenburger Teil.

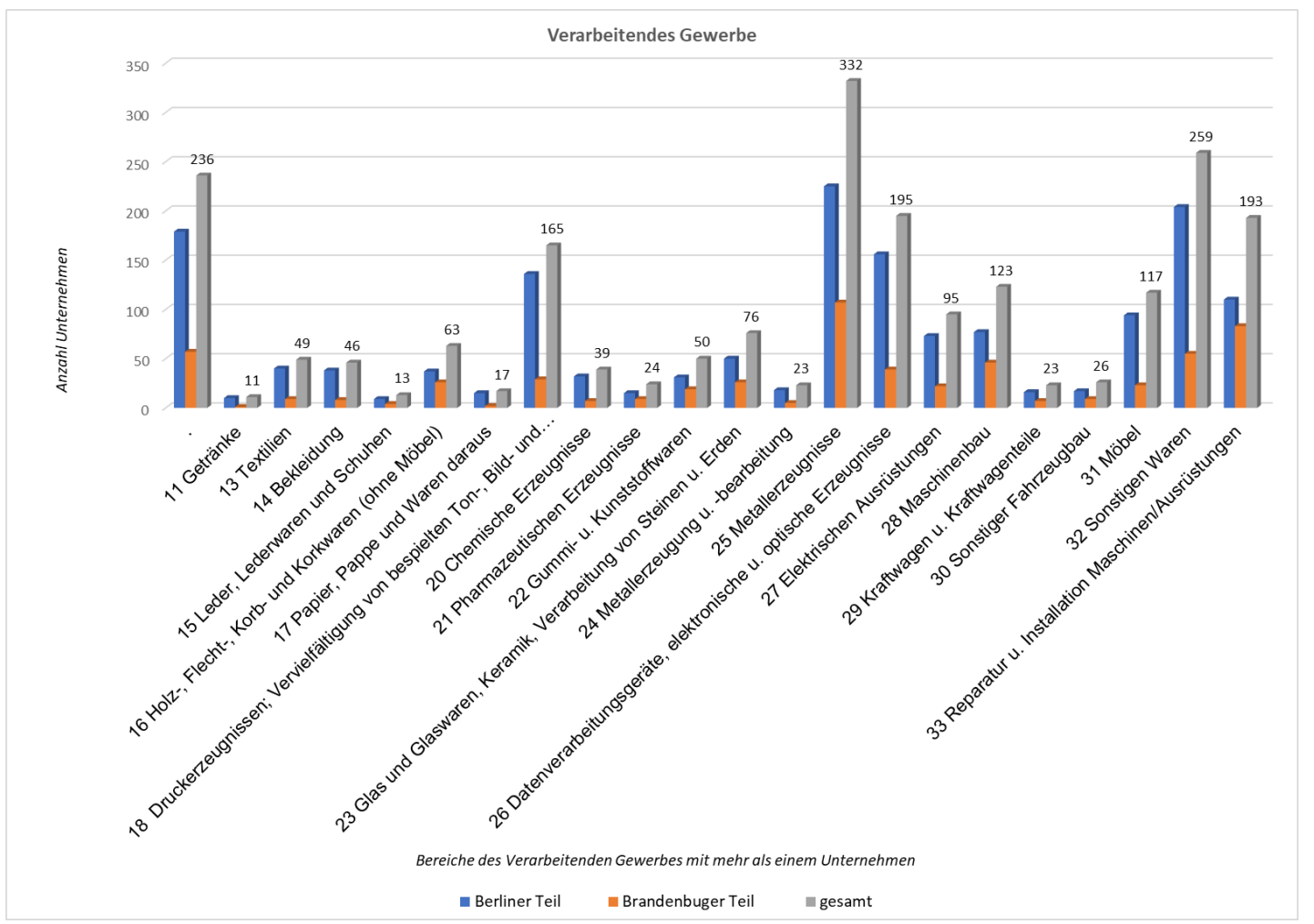

Abbildung 2: Innere Struktur des Verarbeitenden Gewerbes.

Differenziert man das Verarbeitende Gewerbe schließlich räumlich, werden regionale Schwerpunkte erkennbar. In den zur Flughafenregion zählenden Städten und Gemeinden des Landkreises Teltow-Fläming sind es die Bereiche Metall (37 Unternehmen), 
Maschinenbau (20 Unternehmen) und Nahrungs- und Futtermittel (19 Unternehmen), die nach der Anzahl der Unternehmen die ersten Plätze belegen. In den Städten und Gemeinden des Landkreises Dahme-Spreewald entfallen die meisten Unternehmen auf Metallerzeugung (40 Unternehmen), Reparatur und Installation von Maschinen und Anlagen (37 Unternehmen) sowie Nahrungs- und Futtermittel (25 Unternehmen). In den drei Kommunen des Landkreises Oder-Spree ragt die Metallerzeugung heraus (23 Unternehmen) und in der einzigen zur Flughafenregion zählenden Stadt des Landkreises Potsdam Mittelmark (Teltow) dominiert der Bereich der Herstellung von Datenverarbeitungsgeräten. Mit 73 Unternehmen des Verarbeitenden Gewerbes sticht die Stadt Teltow im Brandenburger Teil der Flughafenregion heraus. In allen drei Landkreisen spielt der Bereich Metall eine besondere Rolle. Darüber hinaus ist der Bereich Nahrungsund Futtermittel in den Landkreisen Dahme-Spreewald und Teltow-Fläming dominant. Diese beiden Bereiche gehören allerdings nicht zu den sehr innovativen Bereichen des Verarbeitenden Gewerbes. Dies zeigt sich sowohl am Anteil von Unternehmen mit FuE als auch am Anteil von Unternehmen mit Marktneuheiten (vgl. ZEW 2021: 12-13).

Was den Berliner Teil der Flughafenregion betrifft, so dominieren in TempelhofSchöneberg Metallerzeugnisse (87 Unternehmen), Druckerzeugnisse (75 Unternehmen) und Nahrungs- und Futtermittel (70 Unternehmen). In Neukölln betrifft das vor allem Nahrungs- und Futtermittel (76 Unternehmen) und Metallerzeugnisse (66 Unternehmen), im Stadtbezirk Treptow-Köpenick sind die Bereiche Datenverarbeitungsgeräte (73 Unternehmen) und Metallerzeugnisse (72 Unternehmen) führend.

Neben der Wirtschaftsstruktur ist es die Dynamik im Unternehmensbereich, die einen Eindruck vom Status quo der Flughafenregion vermittelt. Hierfür steht die Gewerbeanzeigenstatistik zur Verfügung, die die Anzahl der Gewerbeanmeldungen und abmeldungen beinhaltet (vgl. StatIS-BBB o. J.). Standorte mit positiver Gewerbeanmeldedynamik in der Flughafenregion sind im Zeitraum 2017-2019 neben allen dreien Berliner Stadtbezirken im Brandenburger Teil insbesondere Königs Wusterhausen, Schönefeld und Ludwigsfelde. Im gleichen Zeitraum waren einige Städte bzw. Gemeinden von einer negativen Gewerbeanmeldedynamik betroffen, wie beispielsweise Fürstenwalde, Lübben oder Luckenwalde. Die Dynamik im Berliner Teil der Flughafenregion ist deutlich größer als die im Brandenburger Teil. Dies zeigt, auch bezüglich der Ansiedlungsdynamik gibt es Unterschiede in der Region.

Die wirtschaftlichen Entwicklungen der Flughafenregion werden auch von den zur Verfügung stehenden Gewerbeflächenpotenzialen konditioniert. Sie sind Voraussetzung für Neuansiedlungen und Unternehmenserweiterungen. In Interviews mit Regionalakteur:innen aus dem Jahr 2020 wurde darauf verwiesen, dass entsprechend dem aktuellen „Gewerbeflächenkonzept Flughafenumfeld BER“ 136 Standorte mit einer Gesamtfläche von 3.941 Hektar vorhanden seien, wovon allerdings bereits 86 Prozent vermarktet sind. Lediglich 27,5 Prozent der Flächen sind im Besitz der öffentlichen Hand, 
was eine gesteuerte Gewerbeflächenentwicklung und -nutzung erschwert. Verfügbar sind nach Abzug der Flächen für den Tesla-Standort in Grünheide (310 Hektar) noch etwa 240 Hektar, wobei es sich um keine großen zusammenhängenden Flächen handelt. Größere und gut erreichbare Flächen finden sich nach Aussage der Experten aus der Wirtschaftsförderung noch in Ragow und Mittenwalde (vgl. Mietzner et al. 2020: 29).

Stellt man die verfügbaren Flächen den prognostizierten Nachfragen im Jahr 2030 gegenüber, wird deutlich, dass die langfristige Deckung des Bedarfs nicht mit den verfügbaren Flächen möglich ist und eine Ausweisung neuer Flächen für Gewerbeansiedlungen notwendig wird (vgl. Braun 2019). Hinzu kommt, dass sich in den letzten Jahren eine weitgehend ungesteuerte Ansiedlung in der Flughafenregion vollzogen hat (ebd.). Mit Ausnahme der Güterverkehrszentren sei, nach Aussagen von Interviewpartnern, keine Profilierung erkennbar. Hiermit im Zusammenhang stünden steigende Immobilienpreise aufgrund der Vermarktungsinteressen privater Eigentümer:innen (vgl. Mietzner et al. 2020: 29).

Neben der Verfügbarkeit von Gewerbeflächen spielt die Verfügbarkeit geeigneter Arbeitskräfte/Fachkräfte für die Wirtschaftsdynamik eine Rolle. Laut Bundesagentur für Arbeit gab es im Dezember 2020 in der Flughafenregion insgesamt 6.083 gemeldete sozialversicherungspflichtige Arbeitsstellen (vgl. Bundesagentur für Arbeit 2021). Die meisten offenen Stellen im Brandenburger Teil der Flughafenregion gab es in Schönefeld und Ludwigsfelde (vgl. Bundesagentur für Arbeit o. J.). Was den Berliner Teil der Flughafenregion betrifft, wurden im Dezember 2020 die meisten offenen Stellen für Tempelhof-Schöneberg (1.295) gemeldet, gefolgt von den Stadtbezirken TreptowKöpenick mit 802 gemeldeten offenen Stellen und Neukölln mit 763 gemeldeten offenen Stellen (ebd.).

Vergleicht man die Anzahl aller gemeldeter Stellen im Dezember 2020 (6.083) mit dem angemeldeten Arbeitskräftebedarf von Tesla, der für den ersten Bauabschnitt auf 12.000 Beschäftigte und für den Endausbau auf 40.000 Beschäftigte geschätzt wird (vgl. Gemeinsame Landesplanungsabteilung Berlin-Brandenburg 2021: 11-12), zeigt sich, welche Dynamik diese Ansiedlung für den Arbeitsmarkt der gesamten Flughafenregion auslöst, mit Konsequenzen für nahezu alle Bereiche der Daseinsvorsorge, insbesondere den Bereich des Wohnens.

Eine wichtige Rolle spielt in diesem Zusammenhang die Möglichkeit, das Potenzial an arbeitslosen Personen der Region für den entstehenden Fachkräftebedarf zu mobilisieren. Darüber hinaus entsteht die Problematik, dass Arbeitskräfte sich auf große und gut bezahlende Unternehmen umorientieren und vor allem KMU der Region sich einem verschärften Fachkräftewettbewerb mit großen Unternehmen gegenübersehen. Einen Hinweis auf vorhandene spezifische Fachkräfteengpässe in der Flughafenregion vermittelt das Fachkräftemonitor von IHK und WFBB des Landes Brandenburg (vgl. IHK/WFBB o. J.) bezogen auf den gesamten IHK Kammerbezirk Cottbus: Hier wurden für 2019 
Fachkräftebedarfsengpässe sowohl in Bezug auf Berufsgruppen als auch Branchen ermittelt. Bei den Berufsgruppen werden Engpässe vor allem in der Gruppe Technische Forschungs-, Entwicklungs-, Konstruktions- und Produktionssteuerungsberufe deutlich. Dies sind genau diejenigen Berufe, die auch von der Tesla-Gigafactory nachgefragt werden dürften. Bei den Branchen finden sich Fachkräfteengpässe besonders im Dienstleistungsbereich, im Baugewerbe sowie im Tourismus (vgl. IHK/WFBB o. J.).

Um die innovativen Kompetenzen der Region zu charakterisieren, wurden in einem ersten Analyseschritt sehr detailliert die innovativen, technologieorientierten Unternehmen im Brandenburger Teil der Flughafenregion identifiziert und Clustern zugeordnet. In einem zweiten Analyseschritt, der auf der Recherche von Webseiten beruhte, wurde das Potenzial innovativer, technologieorientierter Unternehmen für den Berliner Teil der Region abgeschätzt. Anschließend wurden die Unternehmen zusammengenommen Clustern zugeordnet. Die Analyse ergibt folgendes Bild (vgl. Abbildung 3): Ein deutlicher Schwerpunkt ist der Berliner Teil der Flughafenregion, dies insbesondere im Cluster IT, Medien mit knapp 100 Unternehmen. Auch die Cluster Optik und Photonik sowie Energietechnik weisen im Berliner Teil deutlich mehr Unternehmen als im Brandenburger Teil auf.

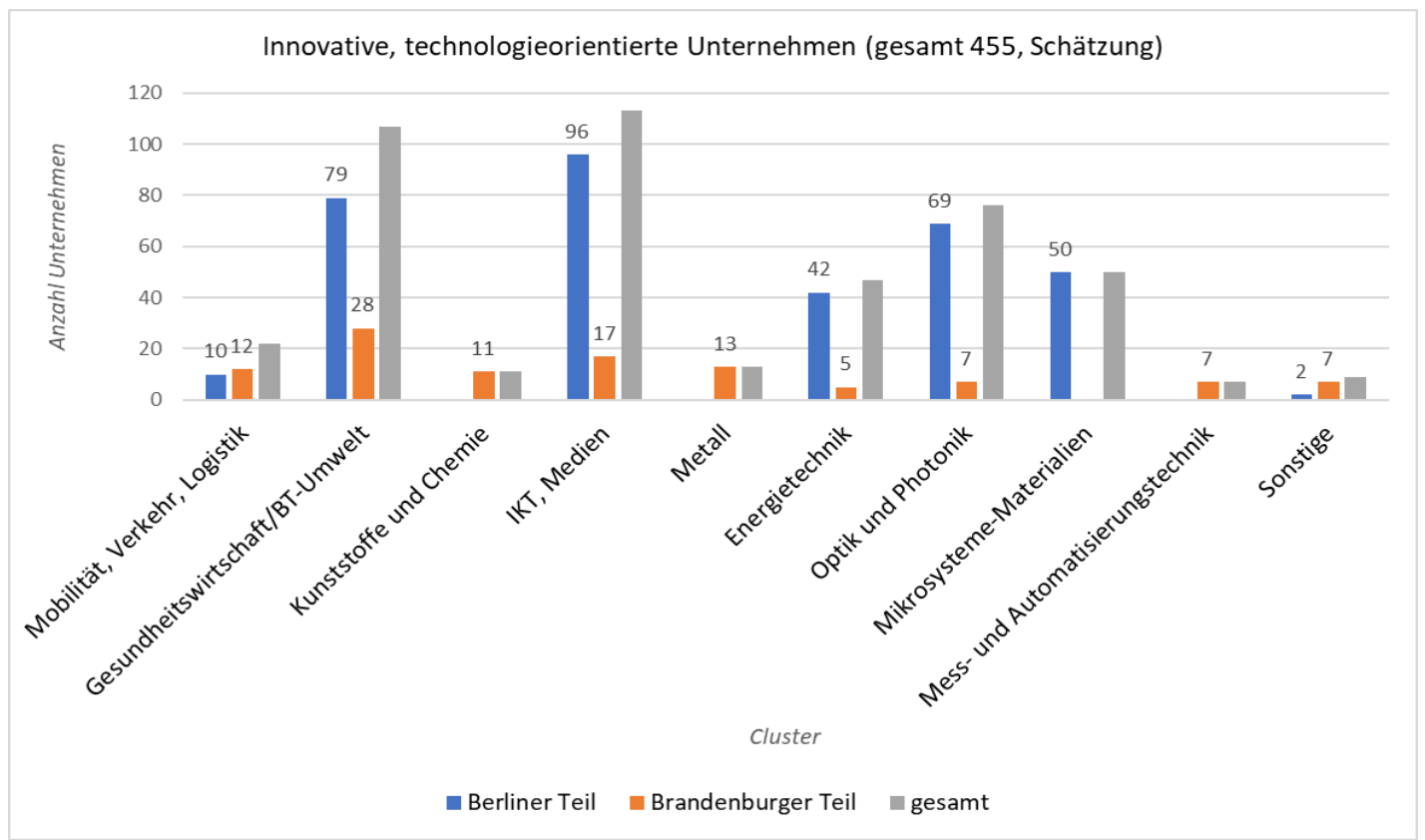

Abbildung 3: Innovative, technologieorientierte Unternehmen. ${ }^{1}$

${ }^{1}$ Für den Berliner Teil: Auf der Basis der Webseiten der Wissenschafts- und Technologiestandorte, Adlershof, Wuhlheide und Spreeknie sowie des EUREF-Campus, zuzüglich der Unternehmen TIB Molbiol, Berlin Chemie und Pepperl+Fuchs. Es handelt sich um Mindestgrößen. Die Cluster Metall sowie Kunststoffe und Chemie sind in Berlin nicht als Cluster geführt. Auch der Bereich Mikrosysteme und Materialien stellt offiziell kein Cluster dar, ebenso wie Messund Automatisierungstechnik. Zusammengefasst wurden die unterschiedlich abgegrenzten Bereiche Gesundheitswirtschaft/Biotechnologie und Umwelt. Für den Brandenburger Teil: Auf der Basis einer Analyse von Förderdatenbanken, 
Relativ stark besetzt sind in beiden Teilen Gesundheitswirtschaft/ BT-Umwelt. In diesem Cluster überwiegen Unternehmen aus den Bereichen Biotechnologie, Pharma und Medizintechnik. Kliniken und Pflegeeinrichtungen sind nicht vertreten. Im Cluster Mobilität, Verkehr, Logistik sind auch Unternehmen aus den Bereichen Luft- und Raumfahrttechnik sowie Automotive vertreten, wenige aus dem übergeordneten Wirtschaftszweig Verkehr und Lagerei.

Die innovativen, technologieorientierten Unternehmen gehören überwiegend dem Wirtschaftszweig des Verarbeitenden Gewerbes an. Ihr Anteil am Verarbeitenden Gewerbe liegt, basierend auf o. g. Schätzung, die von der Mindestanzahl von Unternehmen in den jeweiligen Berliner Zentren ausgeht, im Berliner Teil bei etwa 16 Prozent, im Brandenburger Teil bei etwa 15 Prozent. Die innovativen, technologieorientierten Unternehmen des Clusters IT, Medien, die zu erheblichen Teilen dem Wirtschaftszweig Transport/ Postdienstleistungen, insbesondere den Bereichen Verlage/ Film/ Rundfunk sowie Software/ Informationsdienste/ Telekommunikation zugeordnet werden dürften, wurden herausgerechnet. Das senkt den Anteil innovativer, technologieorientierter Unternehmen am Verarbeitenden Gewerbe besonders im Berliner Teil der Flughafenregion.

Zum Vergleich seien die aktuellen Zahlen zu innovativen Unternehmen in Deutschland aus dem Jahr 2019 angeführt. Diesen Angaben zufolge lag der Anteil von innovativen Unternehmen mit Produktinnovationen im Verarbeitenden Gewerbe im Jahr 2019 bei 33 Prozent, der von innovativen Unternehmen mit gelegentlicher FuE bei 14 Prozent und der von innovativen Unternehmen mit Marktneuheiten bei 12 Prozent (vgl. ZEW 2021: 12-13). $\mathrm{Da}$ die für die Flughafenregion identifizierten Unternehmen in der Regel über FuEAktivitäten identifiziert wurden, liegt ein Vergleich mit dieser Gruppe innovativer Unternehmen nahe und deutet mit 16 Prozent bzw. 15 Prozent auf einen etwas höheren Anteil hin, zumal die Schätzung der Anzahl innovativer, technologieorientierter Unternehmen für den Berliner Teil der Flughafenregion eher konservativ ist.

\subsubsection{Wissenschaftslandschaft und konzentrierte Technologiestandorte}

Die Flughafenregion BER verfügt über eine ausgedehnte und differenzierte Wissenschaftslandschaft. Sie wird durch 13 außeruniversitäre Forschungseinrichtungen, drei Ressortforschungseinrichtungen des Bundes und sieben Hochschulen geprägt. Die überwiegende Zahl von Wissenschaftseinrichtungen (16) befindet sich im Berliner Teil der Flughafenregion. Der größte Standort mit zehn Wissenschaftseinrichtungen ist Adlershof. Im Brandenburger Teil sind sieben Wissenschaftseinrichtungen ansässig.

Diese Wissenschaftseinrichtungen decken ein breites inhaltliches naturwissenschaftlich-

Innovationswettbewerben, Innovationskooperationen sowie Webseiten. Der Bereich Mess- und Automatisierungstechnik wurde zusätzlich eingeführt. Umwelt- und Bioverfahrenstechnik wurde dem Cluster Gesundheitswirtschaft/ BT und Umwelt zugeordnet. 
technisches Spektrum ab, wobei im Bereich der außeruniversitären Forschung physikalische Fachgebiete den Schwerpunkt bilden. Breit naturwissenschaftlich-technisch aufgestellt sind die Hochschule für Technik und Wirtschaft, die Humboldt-Universität zu Berlin und Technische Hochschule Wildau. Die außeruniversitären Forschungseinrichtungen in Adlershof verfügen in ihren Spezialisierungen über ein sehr großes Potenzial an wissenschaftlichen Mitarbeiter:innen. In den Hochschulen der Flughafenregion finden sich auch kultur-, rechts-, sozial- wirtschafts- und verwaltungswissenschaftliche Kompetenzen. Ergänzt werden diese an den Standorten durch Transferstellen, Start-up Services, Acceleratoren oder auch Coworking Spaces. Die TH Wildau betreibt im Brandenburger Teil der Flughafenregion seit einiger Zeit regionale Präsenzstellen in Luckenwalde und Fürstenwalde.

Im Folgenden werden ausgewählte Kompetenzen der Flughafenregion aus dem Wissenschaftsbereich, insofern sie entsprechende Anwendungsbezüge erkennen lassen, Clustern in der Region zugeordnet (vgl. Tabelle 1).

\begin{tabular}{|c|c|c|}
\hline Cluster & Einrichtung & Schwerpunkte \\
\hline \multirow{3}{*}{ 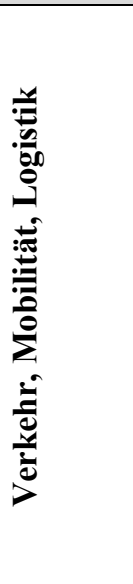 } & $\begin{array}{l}\text { Deutsches Zentrum für } \\
\text { Luft- und Raumfahrt }\end{array}$ & $\begin{array}{l}\text { Verkehrsforschung, Verkehrssystemtechnik, } \\
\text { Fahrzeugkonzepte, optische Sensorsysteme } \\
\text { https://www.dlr.de/content/de/standort/berlin.html }\end{array}$ \\
\hline & $\begin{array}{l}\text { TU-Campus EUREF, } \\
\text { Mobilty2Grid } \\
\text { (EUREF-Campus) }\end{array}$ & $\begin{array}{l}\text { Elektromobilität als Kern einer integrierten } \\
\text { Energieversorgung } \\
\text { https://euref.de/wissenschaft-und-forschung/\#mobility2grid }\end{array}$ \\
\hline & $\begin{array}{l}\text { TH Wildau, } \\
\text { Forschungsfeld } \\
\text { Verkehr und Logistik }\end{array}$ & $\begin{array}{l}\text { Aviation Management /Aviation Security, Luftfahrttechnik } \\
\text { (insbes. unbemannte Kleinflugzeuge), Verkehrslogistik } \\
\text { (insbes. Lagerlogistik, Makrologistik, City Logistik, Logistik } \\
\text { für Forst- und Holzwirtschaft), Sichere Objektidentität } \\
\text { (insbes. Prozess- und Systeminnovation/Industrie 4.0, } \\
\text { Digitalisierung, IoT; Auto ID/RFID-Testzentrum) } \\
\text { https://www.th-wildau.de/forschung-transfer/forschungsfeld-5-verkehr- } \\
\text { und-logistik-transport-and-logistics/ }\end{array}$ \\
\hline \multirow{4}{*}{ 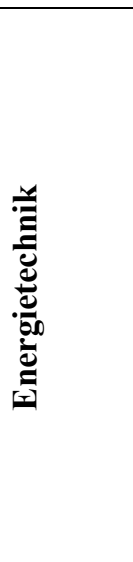 } & $\begin{array}{l}\text { TU-Campus EUREF, } \\
\text { Mobilty2Grid }\end{array}$ & $\begin{array}{l}\text { Soziotechnische Implikationen der Energiewende, } \\
\text { Elektromobilität als Kern einer integrierten } \\
\text { Energieversorgung } \\
\text { https://euref.de/wissenschaft-und-forschung/\#mobility2grid } \\
\end{array}$ \\
\hline & $\begin{array}{l}\text { WindNoDe (EUREF- } \\
\text { Campus) }\end{array}$ & $\begin{array}{l}\text { Verbundprojekt zu regenerativen Energien } \\
\text { https://www.windnode.de/ }\end{array}$ \\
\hline & $\begin{array}{l}\text { Hochschule für } \\
\text { Technik und } \\
\text { Wirtschaft }\end{array}$ & $\begin{array}{l}\text { Forschungsschwerpunkt Regenerative Energie - } \\
\text { Energieeffizienz } \\
\text { https://www.htw-berlin.de/forschung/forschungsprofil// } \\
\text { forschungsschwerpunkte/regenerative-energien-energieeffizienz/ }\end{array}$ \\
\hline & $\begin{array}{l}\text { Helmholtz-Zentrum } \\
\text { für Material und } \\
\text { Energie }\end{array}$ & $\begin{array}{l}\text { Photovoltaik, Materialsysteme für die Energiewende } \\
\text { https://www.helmholtz-berlin.de/forschung/unsere-forschung/ } \\
\text { index_de.html }\end{array}$ \\
\hline 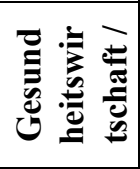 & $\begin{array}{l}\text { Leibniz-Institut für } \\
\text { Analytische } \\
\text { Wissenschaften }\end{array}$ & $\begin{array}{l}\text { Innovative Analytik für die Gesundheitsforschung, } \\
\text { Krankheitsmechanismen und Targets, Biomarker, } \\
\text { Imaging, Biogrenzflächen } \\
\text { https://www.isas.de/forschung }\end{array}$ \\
\hline
\end{tabular}




\begin{tabular}{|c|c|c|}
\hline & $\begin{array}{l}\text { Humboldt Universität, } \\
\text { Institut für Chemie }\end{array}$ & $\begin{array}{l}\text { Chemische Biologie } \\
\text { (u.a. Entwicklung biotechnologischer Werkzeuge) } \\
\text { https://www.chemie.hu-berlin.de/de/forschung/profil }\end{array}$ \\
\hline & $\begin{array}{l}\text { TH Wildau, } \\
\text { Forschungsfeld } \\
\text { angewandte } \\
\text { Biowissenschaften }\end{array}$ & $\begin{array}{l}\text { Bioprozesstechnik, Biosystemtechnik, Molekulare } \\
\text { Biotechnologie und Funktionelle Genomik, } \\
\text { High Performance Computing in Life Sciences, } \\
\text { Diagnostische Bioinformatik, Mikrosystemtechnik/ } \\
\text { Systemintegration } \\
\text { https://www.th-wildau.de/forschung-transfer/forschungsfeld-1-angewandte- } \\
\text { biowissenschaften-applied-life-sciences/ }\end{array}$ \\
\hline & $\begin{array}{l}\text { Hochschule für } \\
\text { Technik und } \\
\text { Wirtschaft }\end{array}$ & $\begin{array}{l}\text { Forschungsschwerpunkt Gesundheit, Biotechnologie, } \\
\text { med. Produkte und Dienstleistungen } \\
\text { https://www.htw-berlin.de/forschung/forschungsprofil/ } \\
\text { forschungsschwerpunkte/gesundheitsforschung/ }\end{array}$ \\
\hline & $\begin{array}{l}\text { Institut für } \\
\text { Biomaterialforschung } \\
\text { (Helmholtz-Zentrum } \\
\text { Geesthacht/Teltow) }\end{array}$ & $\begin{array}{l}\text { Multifunktionale, polymerbasierte Biomaterialien für } \\
\text { Anwendungen in der Regenerativen Medizin } \\
\text { https://www.hereon.de/institutes/active_polymers/index.php.de }\end{array}$ \\
\hline & Robert Koch-Institut & $\begin{array}{l}\text { Zentrum für KI in der Public-Health-Forschung } \\
\text { https://www.rki.de/DE/Content/Institut/OrgEinheiten/Information_ZKI.html }\end{array}$ \\
\hline & $\begin{array}{l}\text { Institut für Gemüse- } \\
\text { und } \\
\text { Zierpflanzenforschung }\end{array}$ & $\begin{array}{l}\text { Pflanzenqualität und Ernährungssicherheit } \\
\text { https://www.igzev.de/ }\end{array}$ \\
\hline \multirow{3}{*}{ 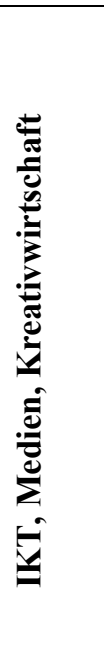 } & $\begin{array}{l}\text { Humboldt-Universität } \\
\text { zu Berlin, Institut für } \\
\text { Informatik }\end{array}$ & $\begin{array}{l}\text { Auswahl: Bild- und Videoanalyse, Datenbanktechnologien, } \\
\text { Drahtlose Breitbandkommunikation, Softwaretechnik, } \\
\text { Adaptive Systeme, Sprachentwicklung, Algorithmisches } \\
\text { Engineering, Data Science und statistische Datenanalyse, } \\
\text { Kommunikationsnetzwerke ... } \\
\text { https://www.informatik.hu-berlin.de/de/forschung/anwendung-forschung }\end{array}$ \\
\hline & $\begin{array}{l}\text { TH Wildau, } \\
\text { Forschungsfeld } \\
\text { Informatik/Telematik }\end{array}$ & $\begin{array}{l}\text { Business Intelligence, Decision Support Systeme, } \\
\text { Gebäudetelematik, Mobile Informations- und } \\
\text { Assistenzsysteme, Softwareentwicklung/ Software } \\
\text { Engineering, } \\
\text { Telematik, Verwaltungsinformatik und digitale Medien } \\
\text { https://www.th-wildau.de/nc/forschung-transferforschungsfeld-2- }\end{array}$ \\
\hline & $\begin{array}{l}\text { Hochschule für } \\
\text { Technik und } \\
\text { Wirtschaft }\end{array}$ & $\begin{array}{l}\text { Forschungsschwerpunkt Digitale Wirtschaft - } \\
\text { Kreativwirtschaft } \\
\text { Digitale Wirtschaft - Kreativwirtschaft (htw-berlin.de) }\end{array}$ \\
\hline$\overline{\bar{\pi}}$ & $\begin{array}{l}\text { TH Wildau, } \\
\text { Forschungsfeld } \\
\text { Produktion und } \\
\text { Material }\end{array}$ & $\begin{array}{l}\text { iC3@Smart Production, Maschinendynamik und lärmarme } \\
\text { Konstruktion sowie Werkstofftechnik, Metallische } \\
\text { Werkstoffe und Analytik } \\
\text { https://www.th-wildau.de/forschung-transfer/forschungsfeld-4-produktion- } \\
\text { und-material-production-and-materials/ }\end{array}$ \\
\hline 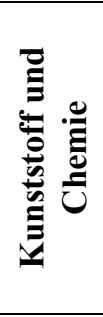 & $\begin{array}{l}\text { TH Wildau, } \\
\text { Forschungsfeld } \\
\text { Produktion und } \\
\text { Material }\end{array}$ & $\begin{array}{l}\text { Forschungsgruppe polymere Hochleistungsmaterialien und } \\
\text { Forschungsgruppe Faserverbund-Materialtechnologien } \\
\text { (Entwicklung von Harzsystemen und Funktionsintegration } \\
\text { bei Compositen) } \\
\text { https://www.th-wildau.de/forschung-transfer/polymere- } \\
\text { hochleistungsmaterialien } \\
\text { https://www.th-wildau.de/forschung-transfer/faserverbund- } \\
\text { materialtechnologien/ }\end{array}$ \\
\hline
\end{tabular}




\begin{tabular}{|c|c|c|}
\hline & $\begin{array}{l}\text { Fraunhofer IAP, FB } \\
\text { Pyco }\end{array}$ & $\begin{array}{l}\text { Forschung zu Polymermaterialien und Composites } \\
\text { https://www.iap.fraunhofer.de/de/Forschungsbereiche/PYCO.html }\end{array}$ \\
\hline \multirow{6}{*}{ 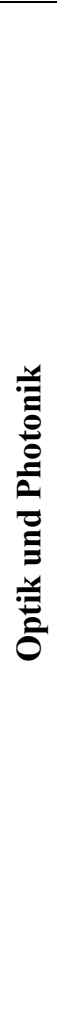 } & $\begin{array}{l}\text { TH Wildau, } \\
\text { Forschungsfeld } \\
\text { Photonik, Laser- und } \\
\text { Plasmatechnologien }\end{array}$ & $\begin{array}{l}\text { Materialsynthese und -untersuchungen, die Herstellung } \\
\text { und Charakterisierung optoelektronischer Bauteile und } \\
\text { Komponenten sowie Prozesscharakterisierung und } \\
\text { Optimierung } \\
\text { https://www.th-wildau.de/forschung-transfer/photonik-laser-und- } \\
\text { plasmatechnologien/ }\end{array}$ \\
\hline & $\begin{array}{l}\text { Max-Born-Institut f. } \\
\text { Nichtlineare Optik u. } \\
\text { Kurzzeitspektroskopie }\end{array}$ & $\begin{array}{l}\text { Nichtlineare Optik und ultraschnelle Dynamik der } \\
\text { Wechselwirkung von Licht mit Materie sowie darauf } \\
\text { basierende Anwendungen, ultraschnelle und intensive Laser } \\
\text { und lasergetriebene Kurzpuls-Lichtquellen in einem breiten } \\
\text { Spektralbereich in Kombination mit Methoden der nicht- } \\
\text { linearen Spektroskopie und zeitaufgelösten } \\
\text { Strukturforschung } \\
\text { https://mbi-berlin.de/research/research-program }\end{array}$ \\
\hline & $\begin{array}{l}\text { Ferdinand Braun } \\
\text { Institut für } \\
\text { Höchstfrequenztechnik }\end{array}$ & $\begin{array}{l}\text { Forschungsbereich Photonik, breites Spektrum an } \\
\text { Entwicklungen zu Diodenlasern und Leuchtdioden (LEDs) } \\
\text { https://www.fbh-berlin.de/forschung/photonik }\end{array}$ \\
\hline & $\begin{array}{l}\text { Humboldt Universität, } \\
\text { FB Physik }\end{array}$ & $\begin{array}{l}\text { Forschungsschwerpunkt Optik/ Photonik } \\
\text { https://www.physik.hu-berlin.de/de/op }\end{array}$ \\
\hline & $\begin{array}{l}\text { DLR, Deutsches } \\
\text { Zentrum für Luft- und } \\
\text { Raumfahrt }\end{array}$ & $\begin{array}{l}\text { Optische Sensorsysteme } \\
\text { https://www.dlr.de/content/de/institutspraesentation/institut-fuer-optische- } \\
\text { sensorsysteme.html }\end{array}$ \\
\hline & OUT e.V. & $\begin{array}{l}\text { Optoelektronik und Sensorik, ... } \\
\text { http://www.out-ev.de/ }\end{array}$ \\
\hline
\end{tabular}

Tabelle 1: Zuordnung wissenschaftlicher Kompetenzen zu Clustern.

Zum Cluster Verkehr, Mobilität, Logistik wird im Themenbereich Verkehr, hier insbesondere Elektromobilität, mit Wissenschaftspotenzialen des DLR in Adlershof und der TU Berlin am Standort EUREF-Campus geforscht. Die Logistik ist an der TH Wildau in den Bereichen Verkehrs- und Produktionslogistik breit aufgestellt. Der Cluster Optik und Photonik ist in Adlershof konzentriert, auch durch die $\mathrm{zu}$ ihm passfähigen Forschungseinrichtungen, ergänzt um Wissenschaftspotenziale in Wildau. Spezielle Kompetenzen mit Bezügen zum Cluster Energietechnik finden sich ebenfalls an den Standorten Adlershof und EUREF-Campus sowie an der Hochschule für Technik und Wirtschaft in Berlin. Der inhaltlich breit gefächerte Cluster Gesundheitswirtschaft/ Biotechnologie-Umwelt kann auf ein ebenso breites Kompetenzspektrum im Wissenschaftsbereich zurückgreifen, das insbesondere in Wildau, Teltow, Adlershof und Schöneweide (HTW) angesiedelt ist. Bezüglich des Clusters Kunststoffe/ Chemie weisen die Wissenschaftseinrichtungen in Teltow und Wildau eine stärkere Spezialisierung im Bereich Polymermaterialien und Composites auf. Der Cluster IKT, Medien, Kreativwirtschaft kann insbesondere auf Wissenschaftspotenziale an der HumboldtUniversität in Adlershof, der TH Wildau und der HTW zurückgreifen.

Der Brandenburger Cluster Metall findet Unterstützungsangebote insbesondere an der TH Wildau im Forschungsfeld Produktion und Material. 
Betrachtet man Wissenschaftseinrichtungen und technologieorientierte Unternehmen zusammen, zeigt sich, dass sich dieses Innovationspotenzial an bestimmten Standorten konzentriert. Den größten Standort und einen wichtigen Treiber in der Flughafenregion bildet Adlershof auch hier mit über 300 innovativen, technologieorientierten Unternehmen und zehn Wissenschaftseinrichtungen. Auf dem EUREF-Campus in TempelhofSchöneberg sind zwei Wissenschaftseinrichtungen und mehr als 20 innovative, technologieorientierte Unternehmen konzentriert, in Wildau drei Wissenschaftseinrichtungen und 22 innovative, technologieorientierte Unternehmen sowie in Teltow 3 Wissenschaftseinrichtungen und 28 innovative, technologieorientierte Unternehmen. Insgesamt sind an diesen Standorten also 17 Wissenschaftseinrichtungen und etwa 372 innovative, technologieorientierte Unternehmen angesiedelt. Das entspricht knapp 80 Prozent des Wissenschaftspotenzials und 82 Prozent des technologieorientierten Innovationspotenzials der Flughafenregion (Erhebungszeiträume August bis November 2020 und Januar bis März 2021).

Insgesamt lässt sich einschätzen, dass in der Flughafenregion eine außergewöhnliche Konzentration an Wissenschaftspotenzialen in räumlicher Nähe besteht, auf die die Unternehmen in den Clustern zurückgreifen können. Damit bestehen hervorragende Voraussetzungen, dass sich diese Cluster in der Zukunft wettbewerbsfähig profilieren können.

\subsubsection{Bevölkerung und Wohnen}

Die Arbeits- und Lebensbedingungen in einer Region sind sowohl Zielgröße als auch Voraussetzung ihrer wettbewerbsfähigen Entwicklung, wobei Wettbewerbsfähigkeit als die Fähigkeit verstanden wird, Unternehmen und Einwohnern eine attraktive und nachhaltige Umwelt zum Leben und Arbeiten zu bieten (vgl. Annoni/Dijkstra 2019: 3). Ein wichtiger Bestandteil dieser regionalen Umwelt sind die Wohnmöglichkeiten, die wiederum andere regionale Entwicklungsbedingungen wie beispielsweise Bildungsangebote und -infrastrukturen, Mobilitätsangebote oder die medizinische Versorgung beeinflussen. Für die Flughafenregion stellen das Angebot und die Nachfrage nach Wohnraum eine besonders wichtige Herausforderung dar. Die Nachfrage nach Wohnraum wird durch das Bevölkerungswachstum beeinflusst, welches als Kriterium der Attraktivität einer Region gewertet werden kann, aber auch mit Herausforderungen verbunden ist. Die mit der Ansiedlung von Tesla erwarteten Folgen für den Zuzug und infolgedessen für die Wohnraumnachfrage verdeutlichen dies exemplarisch (vgl. Gemeinsame Landesplanungsabteilung Berlin-Brandenburg 2021).

Zunächst gilt festzuhalten, dass in allen Kommunen der Flughafenregion und in den Berliner Stadtbezirken in den vergangenen Jahren eine Zunahme der Bevölkerung zu verzeichnen ist (vgl. Statistik-Berlin-Brandenburg 2021a; Statistik-Berlin-Brandenburg 2021b), wodurch sich der Brandenburger Teil der Flughafenregion von vielen anderen 
Teilregionen des Landes Brandenburg unterscheidet, die nach wie vor vom Bevölkerungsrückgang betroffen sind. Der Bevölkerungszuwachs betrug im Berliner Teil der Flughafenregion im Zeitraum 2013-2020 8,3 Prozent und im Brandenburger Teil 8,8 Prozent. Am deutlichsten gewachsen sind in im Zeitraum 2013 bis 2020 die Gemeinde Schönefeld mit 20 Prozent, gefolgt von der Gemeinde Schulzendorf mit 13 Prozent und der Stadt Königs Wusterhausen mit elf Prozent (vgl. Statistik-Berlin-Brandenburg 2021a). Ein Ende des Bevölkerungswachstums ist derzeit nicht in Sicht.

Das Wohnen in der Flughafenregion soll nachfolgend durch die Mietpreisentwicklung und durch das Wohnungsangebot für Mietwohnungen charakterisiert werden. Die Mietpreisentwicklung (Wohnungen bis $80 \mathrm{~m}^{2}$ ) in den zur Flughafenregion zählenden Landkreisen insgesamt zeigt im Zeitraum 2017 bis 2020 einen deutlichen Anstieg. Im Landkreis Dahme-Spreewald insgesamt stiegen die Mietpreise von 7,60 Euro im Jahr 2017 auf 8,90 Euro (17 Prozent) im Jahr 2020, im Landkreis Oder-Spree von 6,30 Euro auf 7,30 Euro (16 Prozent) und im Landkreis Teltow-Fläming von 6,10 Euro auf 7,50 Euro (23 Prozent) (vgl. Immowelt 2021a). Seitdem sind die Mitpreise weiter gestiegen. Sie wiesen im März 2021 eine Spanne von 6,60 Euro und 7,50 Euro in den Regionalen Wachstumskernen Fürstenwalde und Luckenwalde bis zu 12,00 Euro in Ludwigsfelde sowie 12,70 Euro in Wildau und Treptow-Köpenick auf(vgl. Immowelt 2021b). Auffallend sind die hohen Mieten in den zum Landkreis Dahme-Spreewald gehörenden Kommunen der Flughafenregion. Auch Lübben hat inzwischen ein hohes Mietniveau erreicht. Für den Berliner Teil der Region ist die Wirksamkeit des Mietdeckels zu berücksichtigen, der einen weiteren Preisanstieg im Jahr 2020 vermindert haben dürfte, jedoch im April 2021 vom Bundesversfassungsgericht als nichtig erklärt worden ist, so dass in naher Zukunft mit weiter steigenden Mietpreisen zu rechnen ist. Hohe Mietpreise deuten auf die Attraktivität eines Standortes hin, widerspiegeln aber auch die hohe Nachfrage, was auf ein Problem im Bereich des Wohnens hinweist. Für einige Kommunen werden entsprechende Lösungsansätze in Verdichtungsstrategien gesehen. Berlin versuchte, mit dem Mietpreisdeckel den Anstieg der Mieten zu bremsen. Einer stärkeren Besiedlung des Brandenburger Teils der Flughafenregion steht prinzipiell nichts im Wege, so hat die Wohnungsbaupotenzialanalyse im Stadt-Umland Zusammenhang von Complan Kommunalberatung aus dem Jahr 2016 gezeigt, dass entsprechende mittel- und langfristig Baupotenziale zu Verfügung stehen (vgl. Complan Kommunalberatung 2016: 32).

Das ungebrochene Bevölkerungswachstum, das ältere Prognosen für das Jahr 2030 bereits im Jahr 2020 deutlich überstiegen hat (vgl. Jahn, Mack \& Partner 2016: 31, LBV 2015) und dessen Entwicklung im Jahr 2018 modifiziert wurde (vgl. LBV 2018), sowie die Ansiedlung großer Firmen mit erheblichen Arbeitskräftebedarfen dürften das Problem jedoch weiter verschärfen. Eine Handlungsempfehlung aus der Evaluation des Gemeinsamen Strukturkonzeptes bezog sich bereits im Jahr 2016 auf die Entwicklung einer Wohnbauflächenstrategie, einschließlich der Prüfung von Verdichtungspotenzialen und der Identifizierung von geeigneten Standorten für Miet- und Sozialwohnungsbau sowie 
Ansätze zur Überwindung der Entwicklungsengpässe in den Kommunen (vgl. Jahn, Mack \& Partner 2016: 110). Im fortgeschriebenen GSK aus dem Jahr 2021 wird darüber hinaus auf Schwierigkeiten der Mobilisierung von Neubauflächen aufgrund eines hohen Anteiles von Flächen im privaten Eigentum hingewiesen (vgl. Dialogforum Airport BerlinBrandenburg 2021: 19).

Die Effekte der Tesla-Gigafactory auf den Zuzug in die Region (angenommen wird, dass Arbeitskräfte außerhalb einer Pendlerdistanz von 60 Minuten multimodal - ÖPNV/PKW zuziehen), werden für den ersten Bauabschnitt (12.000 Beschäftigte) mit 11.603 Zuzügen und für den zweiten Bauabschnitt (40.000 Beschäftigte) mit 36.394 Zuzügen geschätzt (vgl. Gemeinsame Landesplanung Berlin-Brandenburg 2021: 28). Dementsprechend wird im „Tesla-12.000-Szenario“ mit einer Nachfrage nach Wohnraum in der Größenordnung von 11.000 Wohneinheiten gerechnet (vgl. vgl. Gemeinsame Landesplanung BerlinBrandenburg 2021: 35), zeitlich gestaffelt mit Schwerpunkten im Jahr 2021 und 2022. Damit verschärft sich das Wohnungsproblem in der Flughafenregion weiter.

\section{Diskussion und Fazit}

Die Analyse des Status quo der Flughafenregion BER dient im Rahmen der Szenarioanalyse zur Zukunft der Region dem Verständnis der Beschaffenheit regionaler Entwicklungsbedingung und ihrer Zusammenhänge. Der Schwerpunkt der Analyse lag dabei auf Wirtschaft und Wissenschaft.

Eine sorgfältige empirische Analyse regionaler Entwicklungsbedingungen einer sehr heterogenen Region stößt immer wieder auf Probleme, die mit dem Zuschnitt der Flughafenregion zusammenhängen, der sich aus der Zugehörigkeit einzelner Städte, Gemeinden und Stadtbezirken ergibt. Hinzu kommt deren Zugehörigkeit zu übergeordneten administrativen Strukturen wie Regionale Planungsgemeinschaften, Landkreise oder Bundesländer. Insofern ist es sehr aufwendig und teilweise auch nicht möglich, völlig konsistente statistische Daten für diese spezielle Region zu erheben. Die empirische Analyse bewegte sich dennoch auf einem Differenzierungsniveau, das ein gutes Verständnis des Entwicklungsstandes ermöglicht und die Grundlage bietet, dieses im Rahmen der Szenarioanalyse mit anderer Regionalakteuren zu kommunizieren.

Wie im Abschnitt konzeptionelle Grundlagen (vgl. Anschnitt. 3, S. 4-6) dargestellt, spielen für die Ausprägung von Spezialisierungsmustern die räumliche Nähe von Wissenschaftseinrichtungen und innovativen, technologie-orientierten Unternehmen sowie deren Interaktion ebenso eine Rolle wie die Anbindung der Innovationsaktivitäten an die in der Region vorhandenen Kompetenzen und Strukturen. Diese Innovationsaktivitäten können in der Region entstehen und umgesetzt werden, aber auch durch den Eintritt neuer Player maßgebliche Impulse erhalten. In diesem Zusammenhang hat sich die ursprüngliche 
Annahme bestätigt, dass die Flughafenregion über ein enormes Wissenschaftspotenzial verfügt, auf das die zahlreichen innovativen, technologieorientierten Unternehmen der Region zurückgreifen können. Nach der Anzahl von Unternehmen dominieren die Cluster IKT, Medien, Kreativwirtschaft und Gesundheit (Biotechnologie/ Pharmazie/ Medizintechnik-Umwelt). Kombiniert mit den Kompetenzen im Bereich Wissenschaft spielt der Cluster Photonik und Optik eine herausragende Rolle.

Zugleich werden innerhalb der Flughafenregion konzentrierte Innovationsstandorte sichtbar. Das sind im Berliner Teil der Flughafenregion die Standorte Adlershof, Schöneweide und der EUREF-Campus in Tempelhof-Schöneberg. Im Brandenburger Teil der Flughafenregion spielen insbesondere Wildau/ Zeuthen und Teltow sowie Luckenwalde mit seinem Biotechnologiepark eine wichtige Rolle als regionales Innovationszentrum.

Bei entsprechender sektoraler und regionaler Differenzierung werden Unterschiede in der Wirtschaftsstruktur zwischen dem Berliner und dem Brandenburger Teil der Flughafenregion deutlich. Für den Berliner Teil ist der hohe Anteil des Wirtschaftszweiges „Erbringung von freiberuflichen, wissenschaftlichen und technischen Dienstleistungen“ hervorzuheben. Hier sind auch größere Unternehmen aktiv. Im Brandenburger Teil der Flughafenregion spielt der Wirtschaftszweig „Verkehr und Lagerei“ eine bedeutende Rolle. Allein in Schönefeld sind fünf große Unternehmen aus diesem Bereich angesiedelt. Der Anteil des Verarbeitenden Gewerbes an den Wirtschaftszweigen ist im Brandenburger Teil mit 5,8 Prozent größer als im Berliner Teil mit 3,8 Prozent. Der Anteil innovativer, technologieorientierter Unternehmen am Verarbeitenden Gewerbe liegt etwas über dem deutschen Durchschnitt. Innerhalb des Verarbeitenden Gewerbes spielen die Bereiche „Herstellung von Nahrungs- und Futtermitteln“ sowie „Herstellung von Metallerzeugnissen" eine wichtige Rolle, und zwar in beiden Teilen der Flughafenregion. Im Brandenburger Teil sind immerhin 13 innovative, technologieorientierte Unternehmen dem Cluster Metall zugeordnet, was auf eine entsprechende Passfähigkeit hindeutet. Der Bereich Nahrungs- und Futtermittel findet auf Seiten der innovativen technologieorientierten Unternehmen kaum eine Entsprechung.

Zusammenfassend lässt sich feststellen, dass die Flughafenregion über ein überdurchschnittliches Wissenschaftspotenzial verfügt. In ihr sind relativ viele innovative, technologieorientierte Unternehmen aktiv, die einer Vielzahl von Clustern zugeordnet sind. Sie verfügt jedoch insgesamt über eine heterogene Wirtschaftsstruktur, die bisher kaum Spezialisierungsmuster erkennen lässt.

Bezüglich der eingangs thematisierten Zusammenhänge zwischen regionalen Entwicklungsbedingungen ist die besondere Problemlage des Wohnens hervorzuheben. Sie erfährt ihre Dynamik durch anhaltendes Bevölkerungswachstum, begrenzte Wohnungsbaupotenziale und Schwierigkeiten bei der Mobilisierung von Neubauflächen sowie steigende Mietpreise (siehe aktuell Wegfall des Mietpreisdeckels in Berlin) (vgl. Dialogforum 
Airport Berlin-Brandenburg 2021: 19). Auch die sich abzeichnenden Engpässe bei Gewerbeflächen (vgl. Dialogforum Airport Berlin-Brandenburg 2021: 12) und der Verkehrsinfrastruktur stellen die Region vor neue Herausforderungen. Obwohl die Verkehrsinfrastruktur nicht explizit betrachtet wurde, zeigen sich Probleme insbesondere im motorisierten Individualverkehr mit Berlin-Bezug und bei der Auslastung der Bundesautobahnen sowie der Stadtautobahnen. Nach Spreeplan Verkehr hat das Verkehrsaufkommen insgesamt eine Größenordnung erreicht, die Maßnahmen zur Verkehrsvermeidung oder -verlagerung erforderlich macht (vgl. Spreeplan Verkehr 2019: 63). Was die soziale Infrastruktur betrifft, insbesondere die Versorgung mit Wohnnachfolgeeinrichtungen wie Kitas und Schulen, wird von einem Nachholbedarf ausgegangen, der ebenfalls eine Herausforderung für die Region darstellt (vgl. Dialogforum Airport Berlin-Brandenburg 2021: 26).

Im Rahmen von Vorarbeiten zur Status quo Analyse wurde in Interviews mit verschiedenen Regionalakteur:innen deutlich, dass für die weitere Entwicklung der Flughafenregion nicht nur die Regionalen Wachstumskerne von Bedeutung sind (vgl. Mietzner et al. 2020). In der Diskussion sind Entwicklungsachsen (vgl. Mietzner et al. 2021: 28; Borghorst et al. 2021) entlang des südlichen Berliner Rings, aber auch ausgehend von Berlin nach Luckenwalde und von Berlin nach Lübben. Sie können den Wissensaustausch und die Zusammenarbeit von Einrichtungen und Standorten der Flughafenregion fördern und neue Potenziale für das Wohnen und Arbeiten in der Region eröffnen. Eine Voraussetzung hierfür ist die engere Kooperation zwischen Kommunen, aber auch zwischen den Kommunen, Wirtschaftsförderungen, Wissenschaftseinrichtungen und Unternehmen.

An dieser Stelle sei abschließend auf zwei überraschende Ereignisse hingewiesen, die auf die Entwicklung der Flughafenregion einen maßgeblichen Einfluss haben. Hier ist zunächst die Covid-19-Pandemie zu nennen, die den Flughafen und seine Umfeldregion massiv negativ beeinflusst, darüber hinaus und damit im Zusammenhang auch in der Region ansässige Unternehmen aus dem Bereich der Luftfahrt. Ebenso überraschend, wenn auch eher im positiven Sinn, ist die Ansiedlung eines großen Automobilbauunternehmens mit seiner Gigafactory in der Region. Sie hat maßgebliche Auswirkungen nicht nur auf den regionalen Arbeits- und Wohnungsmarkt oder die Gewerbeflächenverfügbarkeit, sondern auch auf die Wirtschaftsstruktur der Region. Gegebenenfalls besitzt die Ansiedlung das Potenzial zur Ausbildung eines Spezialisierungspfades im Bereich der Elektromobilität. Passfähige Innovationspotenziale dafür wären in der Flughafenregion vorhanden.

Diese überraschenden Ereignisse verweisen nachdrücklich auf mögliche unterschiedliche Zukünfte und damit im Zusammenhang auf die Notwendigkeit des Einsatzes von Foresightmethoden, die sich von Planungs- und Prognosemethoden unterscheiden und diese ergänzen. Eine Szenarioanalyse zur Zukunft der Flughafenregion BER mit partizipativen Ansätzen kommt aus diesem Grund zur rechten Zeit, auch wenn die Umsetzung der partizipativen Ansätze aufgrund der Pandemie nur in digitalen Formaten 
möglich ist. Die vorliegende Status quo Analyse bietet eine geeignete Grundlage dafür, um im Szenarioanalyseprozess zur Zukunft der Flughafenregion als Wissenschafts- und Innovationsstandort systematisch weiter voranzuschreiten.

\section{$\underline{\text { Literaturverzeichnis }}$}

Annoni, Paola/Dijkstra, Lewis (2019): The EU Regional Competitiveness Index 2019. [Online]. Verfügbar unter: https://ec.europa.eu/regional_policy/sources/ docgener/work/2019_03_rci2019.pdf. [letzter Zugriff: 15.02.2021].

Arbeitsagentur (o. J.): Arbeitsmarktberichte 2020. [Online]. Verfügbar unter: https://www.arbeitsagentur.de/vor-ort/rd-bb/arbeitsmarktberichte-2020. [letzter Zugriff: 21.04.2021].

Barjak, Franz (2011): Wissens- und Technologietransfer als Interaktion. Theoretische Überlegungen und Fallbeispiele aus der Schweiz, Bern, Peter Lang AG, Internationaler Verlag der Wissenschaften.

Baum, Herbert/Schneider, Jutta/Esser, Klaus/Kurte, Judith (2005): Wirtschaftliche Effekte des Airports Berlin Brandenburg International. Institut für Verkehrswissenschaft an der Universität zu Köln/KE-Consult Wirtschafts- und Verkehrsberatung im Auftrag der Flughafen Schönefeld GmbH, Köln.

Berlin-Airport (o. J. a): Die Historie des BER im Überblick. [Online]. Verfügbar unter: https://www.berlin-airport.de/de/presse/informationen-ber/rueckblick/historie-berueberblick/index.php. [letzter Zugriff: 31.03.2021].

Berlin-Airport (o. J. b): Verkehrsstatistik. [Online]. Verfügbar unter: https://www.berlinairport.de/de/presse/presseinformationen/ verkehrsstatistik/index.php, https://www.berlin-airport.de/de/presse/presseinformationen/ mitteilungen-archiv/2021/2021-03-12aufsichtsratsitzung/index.php. [letzter Zugriff: 29.03.2021].

Berlin-Airport (o. J. c): Ausbauphasen. [Online]. Verfügbar unter: https://www.berlinairport.de/de/presse/informationen-ber/ausblick/3_ ausbauphasen/index.php. [letzter Zugriff: 29.03.2021].

Berlin-Airport (2021): Flughafengesellschaft setzt auf Teilentschuldung / Aufsichtsrat unterstützt Businessplan zur finanziellen Restrukturierung. Presseinformation vom 12.03.21. [Online]. Verfügbar unter: https://corporate.berlin-airport.de/de/unternehmen-presse/presseportal/pressemitteilungen/2021-03-12-flughafengesellschaft-setztauf-teilentschuldung.html [letzter Zugriff: 30.03.2021].

Borghorst, Hermann/ Freytag, Klaus/Kralinski, Thomas/Stock, Günter (2021): Innovation und Nachhaltigkeit in der Hauptstadtregion. Wie eine modellhafte Strategie für die Achse Berlin-Lausitz aussehen kann? Zukunftsforum Berlin-Brandenburg. Themenge- 
spräch am 4. Juni 2021. [Online]: Verfügbar unter: https://www.stiftungzukunftberlin.eu/fileadmin/szb/daten/Initiativen/Berlin-Forum___Stadtstrategie/Berlin-Brandenburg/Positionspapier_Innovationsachse_Berlin-Lausitz.pdf. [letzter Zugriff: 27.07.2021].

Braun, Nomo (2019): Gewerbeflächenkonzept im Umfeld des Flughafens Berlin-Brandenburg „Willy Brandt“. Fachtagung am 11.09.2019 in Schönefeld. [Online]. Verfügbar unter: https://www.airport-region.de/fileadmin/redaktion/ Praesentationen_GeFlaeko/01_Flashlights_agiplan.pdf. [letzter Zugriff: 13.04.2021].

Bundesagentur für Arbeit (2021): Der Arbeitsmarkt in der Region Berlin-Branden-burg. Monatsbericht Dezember 2020. [Online]. Verfügbar unter: https://www.arbeitsagentur.de/vor-ort/rd-bb/arbeitsmarktberichte-2020. [letzter Zugriff: 14.04.2021].

Bundesagentur für Arbeit (o. J.): Statistik. Einzelausgaben. Gemeldete Arbeitsstellen. [Online]. Verfügbar unter: https://statistik.arbeitsagentur.de/SiteGlobals/Forms/ Suche/Einzelheftsuche_Formular.html?nn=15024\&r_f=bb_OderSpree\&topic_f $=$ gemeldete-arbeitsstellen\&dateOfRevision $=202001-202012$. [letzter Zugriff: 02.07.2021].

Camagni, Roberto/Capello, Roberta (2002): "Milieux Innovateurs and collective learning: from concepts to measurements", in: Acs, Zoltan J./de Groot, Henri L.F./Nijkamp, Peter (Hg.), The emergence of the knowledge economy. A regional perspective, Berlin, Heidelberg \& New York: Spinger, S. 15-45.

Christmann, Gabriele (2015): „Einleitung: Zur kommunikativen Konstruktion von Räumen“, in: Christmann, Gabriele (Hg.), Zur kommunikativen Konstruktion von Räumen. Theoretische Konzepte und empirische Analysen, Wiesbaden: Springer, S. 7-25.

Complan Kommunalberatung (2016): Ermittlung der Wohnungsbaupotenziale im StadtUmland-Zusammenhang von Berlin und Potsdam einschließlich aller Mitglieder des kommunalen Nachbarschaftsforums, S. 1-61.

Cooke, Philip/Uranga, Gomez Mikel/Etxebarria, Goio (1997): "Regional Innovation Systems: organizational and institutional dimensions", in: Research Policy, 26 (4-5), S. 475-491.

Deutsches Patent- und Markenamt (o. J.): Patente 2019. [Online]. Verfügbar unter: https://www.dpma.de/docs/presse/dpma_infografik_patente_dt_2019.pdf. [letzter Zugriff: 02.09.2020].

Dialogforum Airport Berlin-Brandenburg (2021): Fortschreibung des Gemeinsamen Strukturkonzeptes (GSK) Flughafenregion Berlin-Brandenburg 2030. Endbericht, April 2021.

Droß, Michael/Thierstein, Andreas (2011): „Wissensökonomie als Entwicklungstreiber von Flughafenregionen - das Beispiel München“, in: Informationen zur Raumentwicklung, Heft 1.2011, S. 27-36.

Etzkowitz, Henry/Zhou, Chunyan (2008): The triple helix. University-Industry-Government Innovation and Entrepreneurship. London and New York: Routledge. 
Flughafendetails (2021): Größte Flughäfen Europas. [Online]. Verfügbar unter: https://www.flughafendetails.de/fluginfo/groesste-flughaefen-europas. [letzter Zugriff: 20.03.2021].

Foray, Dominique/Goenage, Xabier (2013): The goals of smart specialisation. JRC Scientific and Policy Report. European Commission. S3 Policy Brief Series, No. 01/2013, S. $1-15$.

Foray, Dominique/David, Paul A./Hall, Bronwyn (2009): Smart Specialisation - The Concept. Knowledge Economists Policy Brief $n^{\circ}$ 9, June. European Commission, DG Research, Brussels: S. 1-15. [Online]. Verfügbar unter: https://ec.europa.eu/invest-in-research/pdf/download_en/kfg_policy_brief_no9.pdf. [letzter Zugriff: 31.03.2021].

Gemeinsame Landesplanungsabteilung Berlin-Brandenburg (2021): Landesplanerisches Konzept Entwicklung des Umfeldes der Tesla-Gigafactory Berlin-Branden-burg in Grünheide (Mark). März 2011.

GSK (2007): Gemeinsames Strukturkonzept Flughafenumfeld Berlin Brandenburg International (BBI). Gemeinsame Landesplanungsabteilung Berlin-Brandenburg. Hrsg.: Ministerium für Infrastruktur und Raumordnung und Senatsverwaltung für Stadtentwicklung, S. 1-34.

IAU (2018): Sustainable Airport Areas. Guidelines for Decision Makers. [Online]. Verfügbar unter: https://www.metropolis.org/sites/default/files/resources/2018.03_Sustainable_Airport_Areas_Guidelines_for_decision_makers_0.pdf. [letzter Zugriff: 23.11.2020].

IHK/WFBB (o. J.): Fachkräftemonitor Brandenburg. [Online]. Verfügbar unter: http://www.fkm-brandenburg.de/fachkraeftemonitor.html\#1samUyR, http://www.fkmbrandenburg.de/fachkraeftemonitor.html\#1sftGU1. [letzter Zugriff: 13.04.2021].

Immowelt (2021a): Mietspiegel. [Online]. Verfügbar unter: https://www.immowelt.de/immobilienpreise/landkreis-dahme-spreewald/mietspiegel; https://www.immowelt.de/immobilienpreise/landkreis-teltow-flaeming/mietspiegel; https://www.immowelt.de/immobilienpreise/ landkreis-oder-spree/mietspiegel. [letzter Zugriff: 12.04.2021].

Immowelt (2021b): Mietspiegel. [Online]. Verfügbar unter: Immowelt.de/immobilienpreise/. [letzter Zugriff: 23.03.2021].

Jahn, Mack \& Partner (2016): Gutachten. Evaluierung Gemeinsames Strukturkonzept (GSK) Flughafenumfeld BER, S. 1-129.

Kempton, Lousie/Goddard, John/Edwards, John/Hegyi, Fatime Barbara/Elena-Perez, Susana (2014): Universities and Smart Specialisation. S3 Policy Brief Serie No. 03/2013. European Commission, Joint Research Centre. Institute for Prospective Technological Studies. [Online]. Verfügbar unter: https://publications.jrc.ec. europa.eu/repository/handle/JRC85508. [letzter Zugriff: 26.07.2021].

Koschatzky, Knut (2001): Räumliche Aspekte im Innovationsprozess. Ein Beitrag zur neuen Wirtschaftsgeographie aus Sicht der regionalen Innovationsforschung, MünsterHamburg-London, LIT. 
Kujath, Hans-Joachim/Schmidt, Suntje (2007): Wissensökonomie und die Entwicklung von Städtesystemen. Working Paper, Leibniz-Institut für Regionalentwicklung und Strukturplanung, Erkner, S. 1-32.

LBV (2015): Bevölkerungsvorausschätzung 2014-2030 - Ämter und amtsfreie Gemeinden des Landes Brandenburg, Berichte der Raumbeobachtung - Dezember 2015, S. 153.

LBV (2018): Bevölkerungsvorausschätzung 2017-2030 - Ämter und amtsfreie Gemeinden des Landes Brandenburg, Berichte der Raumbeobachtung - November 2018, S. 153.

Mietzner, Dana/Hartmann, Frank/Melzer, Klaus-Martin/Vossel, Markus/ Bautz, Franziska/Wiechers, Henning (2020): Die Rolle von Wissenschaft und Innovation in der Flughafenumfeldregion: Herausforderungen und Perspektiven. [Online]. Verfügbar unter: https://opus4.kobv.de/opus4-th-wildau/frontdoor/ index/index/ docId/1379. [letzter Zugriff: 08.04.2021].

Munich-Airport (o. J.): Verkehrszahlen. [Online]. Verfügbar unter: https://www.munichairport.de/verkehrszahlen-8850,6 https://de.statista.com/ statistik/daten/studie/226206/umfrage/passagieraufkommen-am-flughafen-london-heathrow/. [letzter Zugriff: 20.03.2021].

Piirainen, Kalle A./Tanner, Anne N./Alkaersig, Lars (2017): "Regional foresight and dynamics of smart specialisation: A typology of regional diversification patterns", in: Technological Forecasting \& Social Change 115 (2017), S. 289-300.

PatBase (о. J.): What is PatBase? [Online]. Verfügbar unter: https://www.patbase. com/. [letzter Zugriff: 17.08.2020].

Porter, Michael E. (1998): Clusters and the new economics of competition. Harvard Business Review 76, no. 6 (November - December 1998). [Online]. Verfügbar unter: https://hbr.org/1998/11/clusters-and-the-new-economics-of-competition. [letzter Zugriff: 14.10 .2020$]$.

Spreeplan Verkehr (2019): Abschlussbericht Grundlagenermittlung Verkehr Flughafenregion BER. Mit Unterstützung durch Jahn, Mack \& Partner Architektur und Stadtplanung, S. 1-116.

StatIS-BBB (о. J.): Unternehmensregister und Gewerbeanzeigen. [Online]. Verfügbar unter: AfS StatIS-BBB - Katalog (statistik-berlin-brandenburg.de). [letzter Zugriff: 02.07.2021].

Statista (2021a): Unternehmen nach Wirtschaftszweigen. [Online]. Verfügbar unter: https:/de.statista.com/statistik/daten/studie/1931/umfrage/unternehmen-nach-wirtschaftszweigen/. [letzter Zugriff: 07.04.2021].

Statista (2021b): Umsatz der Unternehmen in Deutschland nach Wirtschaftszweigen. [Online]. Verfügbar unter: https://de.statista.com/statistik/daten/studie/242084/umfrage/umsatz-der-unternehmen-in-deutschland-nach-wirtschaftszweigen/. [letzter Zugriff: 07.04.2021]. 
Statistik-Berlin-Brandenburg (2021a): Bevölkerungsentwicklung und Bevölkerungsstand im Land Brandenburg Juni 2020. [Online]. Verfügbar unter: https://download.statistikberlin-brandenburg.de/b0e795f290997512/23eaab608505/SB_A01-0700_2020m06_BB.pdf. [letzter Zugriff: 02.07.2021].

Statistik-Berlin-Brandenburg (2021b): Einwohnerinnen und Einwohner im Land Berlin am 31. Dezember 2020. [Online]. Verfügbar unter: https://download.statistik-berlinbrandenburg.de/fa93e3bd19a2e885/a5ecfb2fff6a/SB_A01-05-00_2020h02_BE.pdf. [letzter Zugriff: 02.07.2021].

Statistik der Bundesagentur für Arbeit - Einzelausgaben (o. J.): [Online]. Verfügbar unter: arbeitsagentur.de. [letzter Zugriff: 21.04.2021].

Statistik der Bundesagentur für Arbeit - Einzelausgaben (o. J. a): [Online]. Verfügbar unter: Einzelausgaben - Statistik der Bundesagentur für Arbeit (arbeitsagentur.de). [letzter Zugriff: 21.04.2021].

Vezzani, Antonio/Baccan, Marco/Candu, Alina/Castelli, Alessio/Dosso, Mafini/Gkotsis, Petros M. (2017): Smart Specialisation, seizing new industrial opportunities. JRC Technical Report. European Commission, 2017.

Voß, Rainer (2002): Regionale Innovationssysteme als Gegenstand eines neuen Forschungsverbundes der Region Berlin-Brandenburg, in: Voß, Rainer (Hg.), Regionale Innovationssysteme, Wildauer Schriftenreihe, Band 2, Berlin, Verlag News \& Media, S. 25-46.

Wagner, Dieter/Schultz, Christian (2011): Finanzierung technologieorientierter Unternehmen in Deutschland. Köln: EUL Verlag.

Wiechmann, Thorsten (2000): Die Region ist tot-es lebe die Region! Anmerkungen zur Diskurskonjunktur und Relativierung des Begriffes, in: Raumforschung und Raumordnung | Spatial Research and Planning 58, 173-184 (2000).

WFBB (o. J.): Standort Brandenburg. Branchen und Cluster. [Online]. Verfügbar unter: https://www.wfbb.de/de/Wirtschaftsstandort/Branchen-und-Cluster/. [letzter Zugriff: 13.05.2020].

ZEW (2019). Innovationen in der deutschen Wirtschaft. Indikatorenbericht zur Innovationserhebung 2018. Mannheim 2019. [Online]. Verfügbar unter: http://publica.fraunhofer.de/documents/N-531871.html. [letzter Zugriff: 07.01.2022].

ZEW (2021): Innovationen in der deutschen Wirtschaft. Indikatorenbericht zur Innovationserhebung 2020. Mannheim, März 2021. [Online]. Verfügbar unter: http://ftp.zew.de/pub/zew-docs/mip/20/mip_2020.pdf?v=1616141836:Seite. [letzter Zugriff: 26.03.2021]. 